\title{
Corrugated Surfaces and A.C. Spectrum
}

\author{
Vojkan Jakšić ${ }^{1}$ and Yoram Last ${ }^{2}$ \\ ${ }^{1}$ Department of Mathematics and Statistics \\ University of Ottawa, 585 King Edward Avenue \\ Ottawa, ON, K1N 6N5, Canada \\ ${ }^{2}$ Institute of Mathematics, The Hebrew University \\ Givat Ram, Jerusalem 91904, Israel \\ and \\ Department of Mathematics, Caltech \\ Pasadena, CA 91125, U.S.A.
}

May 31, 1999

\begin{abstract}
We study spectral and scattering properties of the discrete Laplacian $H$ on the half-space $\mathbf{Z}_{+}^{d+1}=\mathbf{Z}^{d} \times \mathbf{Z}_{+}$with boundary condition $\psi(n,-1)=V(n) \psi(n, 0)$. We consider cases where $V$ is a deterministic function and a random process on $\mathbf{Z}^{d}$. Let $H_{0}$ be the Dirichlet Laplacian on $\mathbf{Z}_{+}^{d+1}$. We show that the wave operators $\Omega^{ \pm}\left(H, H_{0}\right)$ exist for all $V$, and in particular, that $\sigma\left(H_{0}\right) \subset \sigma_{\mathrm{ac}}(H)$. We study when and where the wave operators are complete and the spectrum of $H$ is purely absolutely continuous and prove some optimal results. In particular, if $V$ is a random process on a probability space $(\Omega, \mathcal{F}, P)$, such that the random variables $V(n)$ are independent and have densities, we show that the spectrum of $H$ on $\sigma\left(H_{0}\right)$ is purely absolutely continuous $P$-a.s.. If in addition, either $V$ or $V^{-1}$ vanish at infinity, we show that the wave operators $\Omega^{ \pm}\left(H, H_{0}\right)$ are complete on $\sigma\left(H_{0}\right) P$-a.s.
\end{abstract}




\section{Introduction}

This paper deals with spectral and scattering theory of the discrete Laplacian limited to a half-space. The interesting properties of such operators stem from the imposed boundary condition. We refer the reader to $[\mathrm{KP}, \mathrm{JMP}]$ for the history of the problem and to $[\mathrm{AM}, \mathrm{BS}, \mathrm{G}, \mathrm{JM} 1, \mathrm{JM} 2$, JM3, JM4, JMP, KP, M, P] for some recent rigorous work on the subject.

In this section we define the model and state our results.

\subsection{The model}

Let $d \geq 1$ be given, and let $\mathbf{Z}_{+}^{d+1}=\mathbf{Z}^{d} \times \mathbf{Z}_{+}$, where $\mathbf{Z}_{+}=\{0,1, \ldots\}$. We denote the points in $\mathbf{Z}_{+}^{d+1}$ by $(n, x)$, for $n \in \mathbf{Z}^{d}$ and $x \in \mathbf{Z}_{+}$. Let $V: \mathbf{Z}^{d} \mapsto \mathbf{R}$ be a given function and let $H$ be the discrete Laplacian on the Hilbert space $\mathcal{H}:=l^{2}\left(\mathbf{Z}_{+}^{d+1}\right)$ with boundary condition $\psi(n,-1)=V(n) \psi(n, 0)$. When $V=0$, this operator reduces to the Dirichlet Laplacian which we denote by $H_{0}$. The operator $H$ acts as

$$
(H \psi)(n, x)= \begin{cases}\sum_{\left|n-n^{\prime}\right|_{+}+\left|x-x^{\prime}\right|=1} \psi\left(n^{\prime}, x^{\prime}\right) & \text { if } x>0 \\ \psi(n, 1)+\sum_{\left|n-n^{\prime}\right|_{+}=1} \psi\left(n^{\prime}, 0\right)+V(n) \psi(n, 0) & \text { if } x=0,\end{cases}
$$

where $|n|_{+}=\sum_{j=1}^{d}\left|n_{j}\right|$. Physically, the boundary condition $V$ models the corrugated surface of the medium and one is interested in its effects on propagation properties of wave packets.

Note that the operator $H$ can be viewed as the Schrödinger operator

$$
H=H_{0}+V
$$

where the potential $V$ acts only along the surface $\partial \mathbf{Z}_{+}^{d+1}=\mathbf{Z}^{d}$, that is, $(V \psi)(n, x)=0$ if $x>0$ and $(V \psi)(n, 0)=V(n) \psi(n, 0)$. For many purposes it is convenient to adopt this point of view and we will do so in the sequel. Since $H_{0}$ is bounded, the operator $H$ is properly defined as a self-adjoint operator.

We recall that the spectrum of $H_{0}$ is purely absolutely continuous and that

$$
\sigma\left(H_{0}\right)=[-2(d+1), 2(d+1)] .
$$

A simple Weyl's sequence argument yields that for any $V, \sigma\left(H_{0}\right) \subset \sigma(H)$. In this paper we will study spectral and scattering properties of $H$ on the set $\sigma\left(H_{0}\right)$. We will consider the cases where $V$ is a deterministic and a random potential on $\mathbf{Z}^{d}$.

\subsection{Deterministic results}

Unless otherwise stated, all the results of this section hold for an arbitrary surface potential $V$.

The starting point of our work is the following result. 
Theorem 1.1 The wave operators

$$
\Omega^{ \pm}=\mathrm{s}-\lim _{t \rightarrow \mp \infty} \mathrm{e}^{\mathrm{i} t H} \mathrm{e}^{-\mathrm{i} t H_{0}}
$$

exist. In particular, $\sigma\left(H_{0}\right) \subset \sigma_{\mathrm{ac}}(H)$.

Remark 1. Note that the existence of the limits (1.2) implies that for every $s \in \mathbf{R}$,

$$
\mathrm{e}^{\mathrm{i} s H} \Omega^{ \pm}=\Omega^{ \pm} \mathrm{e}^{\mathrm{i} s H_{0}} .
$$

Therefore, $H$ preserves the subspaces $\operatorname{Ran} \Omega^{ \pm}$, and its restrictions to these subspaces are unitarily equivalent to $H_{0}$. This implies that $\sigma\left(H_{0}\right) \subset \sigma_{\text {ac }}(H)$.

Remark 2. After this work was finished we have learned from J. Sahbani that a result similar to Theorem 1.1 has been recently proven in [CS].

Due to the free propagation along the $x$-axis, the above theorem is certainly expected. Somewhat subtler results are related to the question of when and where the wave operators are complete and the spectrum of $H$ is purely absolutely continuous. Before we state our theorems, we need some additional notions.

We decompose $\mathbf{Z}^{d} \times \mathbf{Z}_{+}=\Gamma_{0} \cup \Gamma_{\overline{0}}$, where $\Gamma_{0}=\mathbf{Z}^{d} \times\{0\}$ and $\Gamma_{\overline{0}}=\mathbf{Z}^{d} \times\{1,2, \ldots\}$. Let

$$
\mathcal{H}^{\mathrm{s}}:=l^{2}\left(\Gamma_{0}\right) \cong l^{2}\left(\mathbf{Z}^{d}\right), \quad \mathcal{H}^{\mathrm{b}}:=l^{2}\left(\Gamma_{\overline{0}}\right) .
$$

We will refer to these Hilbert spaces as the surface and the bulk. Clearly,

$$
\mathcal{H}=\mathcal{H}^{\mathrm{s}} \oplus \mathcal{H}^{\mathrm{b}} \text {. }
$$

With respect to this decomposition the operator $H$ can be written as a $2 \times 2$ matrix,

$$
H=\left[\begin{array}{ll}
H^{\mathrm{ss}} & H^{\mathrm{sb}} \\
H^{\mathrm{bs}} & H^{\mathrm{bb}}
\end{array}\right]
$$

Here, $H^{\mathrm{ss}}=h_{0}+V$, where $h_{0}$ is the usual discrete Laplacian on $l^{2}\left(\mathbf{Z}^{d}\right)$, and $H^{\mathrm{bb}}$ is the Dirichlet Laplacian on $l^{2}\left(\Gamma_{\overline{0}}\right)$. Note that $H^{\mathrm{bb}}$ is unitarily equivalent to $H_{0}$. The operators $H^{\mathrm{sb}}$ and $H^{\mathrm{bs}}$ couple the surface and the bulk. Clearly, $H^{\mathrm{sb}}=\left(H^{\mathrm{bs}}\right)^{*}, H^{\mathrm{bs}} \delta_{(n, x)}=0$ if $x>0$ and $\left.H^{\mathrm{bs}} \delta_{(n, 0}\right)=\delta_{(n, 1)}$.

We will use notation similar to (1.5) for other operators on $\mathcal{H}$. For example, $\mathbf{1}^{\text {ss }}$ is the projection onto the surface and $\mathbf{1}^{\mathrm{bb}}$ is the projection onto the bulk.

For any $z \notin \sigma\left(H^{\mathrm{bb}}\right)$, we define

$$
\begin{aligned}
& W_{\mathrm{s}}(z):=H^{\mathrm{sb}}\left(H^{\mathrm{bb}}-z \mathbf{1}^{\mathrm{bb}}\right)^{-1} H^{\mathrm{bs}}, \\
& G_{\mathrm{s}}(z):=H^{\mathrm{ss}}-W_{\mathrm{s}}(z)-z \mathbf{1}^{\mathrm{ss}}
\end{aligned}
$$

In physics literature, the operator $W_{\mathrm{s}}(z)$ is sometimes called the self-energy. Following [DJ], we will call $G_{\mathrm{s}}(z)$ the resonance function. Its importance stems from the identity

$$
\mathbf{1}^{\mathrm{ss}}(H-z)^{-1} \mathbf{1}^{\mathrm{ss}}=G_{\mathrm{s}}^{-1}(z)
$$


which is a consequence of the Feshbach formula for $(H-z)^{-1}$ (see Section 2.2). One can also show that the Hilbert space $\mathcal{H}^{\mathrm{s}}$ is a cyclic set for $H$ (see Section 3.1 ). It then follows from Relation (1.7) that the spectral properties of $H$ are encoded by the resonance function $G_{\mathrm{s}}(z)$.

We remark that the objects (1.6) appear naturally in many different problems in mathematical physics. In particular, they have been recently used in [DJ] in the study of some Hamiltonians of quantum field theory. Our notation and some of our abstract results in Section 2 are motivated by [DJ].

In the sequel, we will use the short-hand $\mathbf{C}_{ \pm}:=\{z: \pm \operatorname{Im} z>0\}$. We will show in Section 3.1 that the operator-valued function $\mathbf{C}_{+} \ni z \mapsto W_{\mathrm{s}}(z)$ extends by continuity to a norm-continuous function on $\overline{\mathbf{C}}_{+}$. Thus, for $e \in \mathbf{R}$, we can define $G_{\mathrm{s}}(e)$ by (1.6). Clearly, $G_{\mathrm{s}}(e)$ is a closed operator with domain $\mathcal{D}(V)$. We define the resonant spectrum of the operator $H$ by

$$
\mathfrak{R}(H):=\left\{e \in \mathbf{R}: 0 \in \sigma\left(G_{\mathrm{s}}(e)\right)\right\} .
$$

The basic properties of this set are summarized in

\section{Proposition 1.2}

(i) $\mathfrak{R}(H)$ is a closed set.

(ii) $\mathfrak{R}(H) \subset \sigma(H)$.

(iii) $\sigma(H) \backslash \sigma\left(H_{0}\right) \subset \mathfrak{R}(H)$.

The existence of the resonant spectrum is linked to the surface potential $V$. For example, we will show that if $\|V\|<1$ then $\mathfrak{R}(H)=\emptyset$.

We now recall some basic notions of scattering theory. Let $A$ and $B$ be self-adjoint operators on a Hilbert space $\mathfrak{H}$ and assume that the wave operators

$$
U^{ \pm}:=\mathrm{s}-\lim _{t \rightarrow \mp \infty} \mathrm{e}^{\mathrm{i} t B} \mathrm{e}^{-\mathrm{i} t A} \mathbf{1}_{\Theta}(A)
$$

exist. The relation $\mathrm{e}^{\mathrm{i} s B} U^{ \pm}=U^{ \pm} \mathrm{e}^{\mathrm{i} s A}$ yields that for any bounded Borel function $f$, $f(B) U^{ \pm}=U^{ \pm} f(B)$, and in particular that $\operatorname{Ran} U^{ \pm} \subset \operatorname{Ran} \mathbf{1}_{\Theta}(B)$. The wave operators $U^{ \pm}$are complete on $\Theta$ if $\operatorname{Ran} U^{ \pm}=\operatorname{Ran} \mathbf{1}_{\Theta}(B)$. If $\Theta=\mathbf{R}$, we simply say that the wave operators $U^{ \pm}$are complete. The wave operators $U^{ \pm}$are complete on $\Theta$ iff the wave operators

$$
W^{ \pm}:=\mathrm{s}-\lim _{t \rightarrow \mp \infty} \mathrm{e}^{\mathrm{i} t A} \mathrm{e}^{-\mathrm{i} t B} \mathbf{1}_{\Theta}(B)
$$

exist. If the wave operators are complete on $\Theta$ and $\Theta^{\prime}$ is such that $\operatorname{Ran} \mathbf{1}_{\Theta^{\prime}}(H) \subset$ $\operatorname{Ran} \mathbf{1}_{\Theta}(H)$, then the wave operators are also complete on $\Theta^{\prime}$.

Recall that the wave operators $\Omega^{ \pm}$are given by (1.2). Our next result is

Theorem 1.3 The wave operators $\Omega^{ \pm}$are complete on $\sigma(H) \backslash \mathfrak{R}(H)$. In particular, the spectrum of $H$ on this set is purely absolutely continuous. 
Remark. There are examples of surface potentials $V$ (which even vanish at infinity) such that $H$ has eigenvalues in $\mathfrak{R}(H) \cap \sigma\left(H_{0}\right)$ [MV1]. It is likely that these examples can be modified to produce potentials $V$ for which $\mathfrak{R}(H) \cap \sigma\left(H_{0}\right) \neq \emptyset$ and $\sigma_{\mathrm{pp}}(H)=\mathfrak{R}(H)$ [N, S1, MV2]. Thus, we believe that Theorem 1.3 is an optimal result in the sense that it holds for an arbitrary surface potential $V$.

We proceed to obtain some information on the location of the set $\mathfrak{R}(H)$. In the sequel, if $X, Y \subset \mathbf{R}$, we write $X+Y=\{x+y: x \in X, y \in Y\}$. If either $X=\emptyset$ or $Y=\emptyset$, we set $X+Y=\emptyset$. If $V$ is a constant surface potential, $V=a$, then $\mathfrak{R}(H)=\emptyset$ if $|a|<1$ and

$$
\mathfrak{R}(H)=[-2 d, 2 d]+\left\{a+a^{-1}\right\},
$$

if $|a| \geq 1$. Motivated by this observation, we set

$$
\mathcal{S}(V):=[-2 d, 2 d]+\left\{a+a^{-1}: a \in \sigma(V),|a| \geq 1\right\} .
$$

We also define

$$
\mathcal{S}_{\text {ext }}(V):=\mathcal{S}(V) \cup([-2 d, 2 d]+\{2 a: a \in \sigma(V),|a|<1\}) .
$$

Here, $\sigma(V)=\overline{\left\{V(n): n \in \mathbf{Z}^{d}\right\}}$. Note that if $\inf _{n}|V(n)| \geq 1$, then $\mathcal{S}(V)=\mathcal{S}_{\text {ext }}(V)$.

\section{Theorem 1.4}

(i) $\sigma(H) \subset \sigma\left(H_{0}\right) \cup \mathcal{S}(V)$.

(ii) $\mathfrak{R}(H) \subset \mathcal{S}_{\text {ext }}(V)$.

(iii) If $\|V\|<1$, then $\mathfrak{R}(H)=\emptyset$.

This result and Theorem 1.3 imply that on the set $\sigma(H) \backslash \mathcal{S}_{\text {ext }}(V)$, the spectrum of $H$ is purely absolutely continuous and the wave operators $\Omega^{ \pm}$are complete. If $\|V\|<1$, the operator $H$ has only absolutely continuous spectrum and the wave operators are complete.

It appears difficult to say substantially more about spectral theory of $H$ without making some further assumptions on the model. For example, one can proceed by assuming that $V$ has suitable decay or growth properties and we will briefly pursue this direction in Section 3.4. The case where $V$ is the Maryland potential has been studied in [KP, JM1, JM4]. In the next section, we will consider the case where $V$ is a random process on $\mathbf{Z}^{d}$. This case is of particular physical importance.

\subsection{Random boundary condition}

Let us describe the random surface model we will study. Let $\Omega$ be the set of all surface potentials, that is, the functions $V: \mathbf{Z}^{d} \mapsto \mathbf{R}$. The set $\Omega$ can be identified with

$$
\Omega=\mathbf{R}^{\mathbf{Z}^{d}}=\underset{\mathbf{Z}^{d}}{X} \mathbf{R} .
$$


Let $\mathcal{F}$ be the $\sigma$-algebra in $\Omega$ generated by the cylinder sets $\left\{V: V\left(n_{1}\right) \in B_{1}, \ldots, V\left(n_{k}\right) \in\right.$ $\left.B_{k}\right\}$, where $B_{1}, \ldots, B_{k}$ are Borel subsets of $\mathbf{R}$. The model is specified by the choice of probability measure $P$ on $(\Omega, \mathcal{F})$.

In this work we will consider measures $P$ of the form

$$
P=\underset{n \in \mathbf{Z}^{d}}{\chi} \mu_{n}
$$

where each $\mu_{n}$ is a probability measure on $\mathbf{R}$. Note that $\mu_{n}$ is the probability distribution of the random variable $\Omega \ni V \mapsto V(n)$. We say that the random variable $V(n)$ has density, if the measure $\mu_{n}$ is absolutely continuous w.r.t. Lebesgue measure. Obviously, the random variables $\{V(n)\}$ are independent, and we say that they are i.i.d. if all the measures $\mu_{n}$ are equal to $\mu$. We recall that the topological support of $\mu, \operatorname{supp} \mu$, is the complement of the largest open set $B$ such that $\mu(B)=0$. We set

$$
\mathcal{S}:=[-2 d, 2 d]+\left\{a+a^{-1}: a \in \operatorname{supp} \mu,|a| \geq 1\right\} .
$$

As usual in the theory of random Schrödinger operators, we are interested in the spectral properties of $H=H_{0}+V$ which hold $P$-a.s., that is, for a set of $V$ 's of $P$ measure 1 .

We discuss first the i.i.d. case. Note that if $T_{j}$ is the shift operator on the probability space $\Omega,\left(T_{j} V\right)(n)=V(n-j)$, and $\left(U_{j} \psi\right)(n, x)=\psi(n-j, x)$, then

$$
U_{j} H U_{j}^{*}=H_{0}+T_{j} V
$$

Therefore, $H=H_{0}+V$ is an ergodic family of random operators and it follows from the well-known argument (see [CFKS] for details) that there exist closed sets $\Sigma, \Sigma_{\mathrm{ac}}, \Sigma_{\mathrm{pp}}$, $\Sigma_{\mathrm{sc}}$ such that $P$-a.s., $\sigma(H)=\Sigma, \sigma_{\mathrm{ac}}(H)=\Sigma_{\mathrm{ac}}, \sigma_{\mathrm{pp}}(H)=\Sigma_{\mathrm{pp}}, \sigma_{\mathrm{sc}}(H)=\Sigma_{\mathrm{sc}}$. Obviously, $\Sigma=\Sigma_{\mathrm{ac}} \cup \Sigma_{\mathrm{pp}} \cup \Sigma_{\mathrm{sc}}$. Note that $P$-a.s., $\sigma(V)=\operatorname{supp} \mu$. Thus,

$$
\mathcal{S}(V)=\mathcal{S} \quad P-\text { a.s. }
$$

We can now complement Theorem 1.4 with the following result.

Theorem 1.5 Assume that the random variables $\{V(n)\}_{n \in \mathbf{Z}^{d}}$ are i.i.d. Then,

(i) $\Sigma=\sigma\left(H_{0}\right) \cup \mathcal{S}$.

(ii) There is a set $\mathfrak{R}$ such that $\mathfrak{R}(H)=\mathfrak{R} P$-a.s. Moreover, $\mathcal{S} \subset \mathfrak{R}$.

Remark. Although Part (i) of this theorem is known to workers in the field, its complete proof is not available in the literature. For completeness, we include the proof here.

Combining Theorems 1.4 and 1.5, we show that if $\operatorname{supp} \mu \cap(-1,1)=\emptyset$, then $\mathfrak{R}(H)=\mathcal{S}$ $P$-a.s.. Of course, if $\operatorname{supp} \mu \subset(-1,1)$, then $\mathfrak{R}(H)=\emptyset P$-a.s..

Our next result concerns the structure of the spectrum of $H$ in $\sigma\left(H_{0}\right)$. As we have already discussed, it is known that for some surface potentials $V, H$ may have embedded 
eigenvalues in $\sigma\left(H_{0}\right)$. The general wisdom, however, suggests that a singular spectrum embedded in an absolutely continuous spectrum is unstable with respect to "generic perturbations". In the random case, the vague notion of "generic" could be replaced with a precise $P$-a.s. statement, and a natural conjecture is that $P$-a.s. the spectrum of $H$ in $\sigma\left(H_{0}\right)$ is purely absolutely continuous. This is indeed the context of our next and perhaps deepest result.

Theorem 1.6 Assume that the random variables $\{V(n)\}_{n \in \mathbf{Z}^{d}}$ have densities. Then, the spectrum of $H$ in $\sigma\left(H_{0}\right)$ is $P$-a.s. purely absolutely continuous.

Remark 1. Independence of $\{V(n)\}$ is not needed. It suffices that for all $n$ the conditional distribution of $V(n)$, conditioned on $\{V(m)\}_{m \neq n}$, has density.

Remark 2. This theorem is a special case of a general result concerning the structure of the spectrum of Anderson-type models recently proven by the authors in [JL1].

Remark 3. The proof of Theorem 1.6 gives some additional information. For example, from our arguments it follows that the spectrum of $H$ in $\sigma\left(H_{0}\right)$ is $P$-a.s. transient absolutely continuous in the sense of Avron-Simon [AS].

Our final result deals with the case where either $V$ or $V^{-1}$ vanishes at infinity.

Theorem 1.7 Assume that the assumption of Theorem 1.6 holds, and that either

$$
\lim _{|n| \rightarrow \infty} V(n)=0 P-a . s . \quad \text { or } \quad \lim _{|n| \rightarrow \infty}|V(n)|=\infty P-a . s .
$$

Then,

(i) The resonant spectrum of $H$ has Lebesgue measure zero P-a.s.

(ii) The wave operators $\Omega^{ \pm}$are complete on $\sigma\left(H_{0}\right) P$-a.s.

Remark. Under the assumptions (1.12), the spectrum of $H$ outside $\sigma\left(H_{0}\right)$ is $P$-a.s. discrete. See Section 3.4.

It is interesting to compare Theorem 1.6 with some recent results about localization for $H$ proven in [JM2, JM3]. For simplicity, we will consider only the i.i.d. case. Let $\mathrm{d} \mu=p(x) \mathrm{d} x$. It is convenient to introduce a "disorder" parameter $\lambda$, that is, to consider the family of operators

$$
H_{\lambda}=H_{0}+\lambda V
$$

We set $\Sigma_{\mathrm{c}}=\Sigma_{\mathrm{ac}} \cup \Sigma_{\mathrm{sc}}$. If $d=1, p \in L^{\infty}(\mathbf{R})$ and the topological boundary of $\operatorname{supp} \mu$ is a discrete set, it was shown in [JM2] that for all $\lambda$

$$
\Sigma_{\mathrm{c}} \cap\{e:|e|>4\}=\emptyset .
$$

In [JM3] it was shown that for any $d$ there are constants $\lambda_{d}$ and $\Lambda_{d}$, which depend only on $d$ and the density $p$, such that for $|\lambda|<\lambda_{d}$ and $|\lambda|>\Lambda_{d}$

$$
\Sigma_{\mathrm{c}} \cap\{e:|e|>2(d+1)\}=\emptyset
$$


(weaker results are proven in $[\mathrm{AM}, \mathrm{G}]$ ). The conditions on the density $p$ for which this result hold are the same as in [AM]. Thus, whenever (1.13) or (1.14) hold, it follows from Theorem 1.6 that

$$
\Sigma_{\mathrm{ac}}=\sigma\left(H_{0}\right), \quad \Sigma_{\mathrm{sc}}=\emptyset
$$

and

$$
\Sigma_{\mathrm{pp}}=\overline{\Sigma \backslash \sigma\left(H_{0}\right)}=\overline{\mathcal{S} \backslash \sigma\left(H_{0}\right)}
$$

\subsection{Some remarks}

The spectral theory of random operators $H=H_{0}+V$ on the set $\mathbf{R} \backslash \sigma\left(H_{0}\right)$ is reasonably well understood. The remaining open problems in this direction are closely linked to some fundamental open problems in the theory of random Schrödinger operators. For example, the question whether $H$ has some absolutely continuous spectrum outside $\sigma\left(H_{0}\right)$ is related to the phenomenon of Anderson delocalization (see [JM3]).

In this paper we have concentrated on a much less studied subject, namely, the spectral theory of $H$ on the set $\sigma\left(H_{0}\right)$. Our goal has been to understand spectral and scattering properties of $H$ which follow essentially from the structure of the model and are insensitive to details of the surface potential. It is perhaps surprising how far one can go in that direction using only relatively simple operator-theoretic means. For example, if $V$ is a random process, Theorem 1.6 gives complete characterization of the structure of the spectrum in the set $\sigma\left(H_{0}\right)$.

For reasons of space, in this paper we have restricted ourselves to an essentially timeindependent approach to the spectral and scattering theory of the model (1.1). The dynamical aspects of the model will be discussed in the continuation of this paper [JL2].

The paper is organized as follows. In Section 2, we extract the key structural properties of the model (1.1) and prove Proposition 1.2 and Theorem 1.3 in an abstract framework. In fact, practically all the results of our paper follow from these few structural properties and can be cast into an abstract form. In Section 2, we illustrate how that could be done. Besides (1.1), there are many other "surface type" models which appear in physics (see e.g. $[\mathrm{KP}, \mathrm{JMP}])$ and the results of Section 2 indicate how the techniques of our paper can be used in the study of such models. The basic tools used in Section 2 are Kato's theory of smooth perturbations and the Feshbach formula.

In Section 3, we verify the assumptions of the abstract approach of Section 2 and establish the results described in Section 1.2. In Section 3.4, we study surface potentials which vanish at infinity.

Random surface potentials are studied in Sections 4 and 5. In Section 4, we prove Theorem 1.5. In Section 5, we prove Theorems 1.6 and 1.7.

Our final remark concerns some technical aspects of the paper. Our arguments are based on techniques of scattering theory and the theory of random Schrödinger operators and, it is likely that future developments will also be based on the fusion of techniques of 
these two fields. Since these techniques are rarely combined together, we have attempted to make the paper essentially self-contained.

Acknowledgements. We are grateful to J. Derezinski, S. Molchanov, L. Pastur, J. Sahbani and B. Simon for useful discussions. In particular, we are grateful to B. Simon for convincing us at an early stage that Theorem 1.6 must hold, and to J. Sahbani for indicating to us some simplifications of our original arguments. This work was partially supported by NATO Collaborative Research Grant CRG 970051. VJ's work was also partially supported by NSERC. YL's work was also partially supported by NSF grant DMS-9801474 and by an Allon fellowship.

\section{Abstract framework}

\subsection{Smooth perturbations}

Let $V$ be closed and $A$ a self-adjoint operator on a Hilbert space $\mathfrak{H}$. We say that $V$ is $A$-smooth if $\mathcal{D}(A) \subset \mathcal{D}(V)$ and

$$
\sup _{z \notin \mathbf{R}}|\operatorname{Im} z|\left\|V(A-z)^{-1}\right\|^{2}<\infty .
$$

The condition (2.17) has a number of equivalent reformulations. The one which will concern us below is that $V$ is $A$-smooth iff there exist $C>0$ such that for all $\psi \in \mathfrak{H}$,

$$
\int_{\mathbf{R}}\left\|V \mathrm{e}^{-\mathrm{i} t A} \psi\right\|^{2} \mathrm{~d} t \leq C\|\psi\|^{2}
$$

Let $\Theta \subset \mathbf{R}$ be a Borel set. We denote by $\mathbf{1}_{\Theta}(A)$ the spectral projection of $\mathrm{A}$ onto $\Theta$. We say that $V$ is $A$-smooth on $\Theta$ iff $V \mathbf{1}_{\Theta}(A)$ is $A$-smooth. One can show (see Theorem XIII.30 in [RS2]) that $V$ is $A$-smooth on $\Theta$ if $\mathcal{D}(A) \subset \mathcal{D}(V)$ and

$$
\sup _{e \in \Theta, y>0}\left\|V(A-e-\mathrm{i} y)^{-1} V^{*}\right\|<\infty .
$$

Now let $\mathfrak{H}$ be a Hilbert space decomposed into direct sum $\mathfrak{H}=\mathfrak{H}^{\mathrm{s}} \oplus \mathfrak{H}^{\mathrm{b}}$. We will refer to the Hilbert space $\mathfrak{H}^{\mathrm{s}}$ as the surface and to $\mathfrak{H}^{\mathrm{b}}$ as the bulk. The projections onto $\mathfrak{H}^{\mathrm{s}}$ and $\mathfrak{H}^{\mathrm{b}}$ we denote by $\mathbf{1}^{\mathrm{ss}}$ and $\mathbf{1}^{\mathrm{bb}}$.

We now describe the main result of this section which reduces the proof of Theorem 1.3 to a number of easily verifiable conditions.

Theorem 2.1 Let $H$ and $H_{0}$ be self-adjoint operators on $\mathfrak{H}$ and let $\Theta$ be a bounded open interval. Assume that

(a) $\mathbf{1}^{\text {ss }}$ is both $H$ and $H_{0}$-smooth on $\Theta$. 
(b) $H \mathbf{1}^{\mathrm{bb}}-\mathbf{1}^{\mathrm{bb}} H_{0}=V T V$, where $T$ is a bounded operator, $V$ is bounded and self-adjoint, and $V$ is both $H$ and $H_{0}$-smooth on $\Theta$.

(c) The operators $\left[H, \mathbf{1}^{\mathrm{ss}}\right],\left[H_{0}, \mathbf{1}^{\mathrm{ss}}\right]$ are bounded.

Then the wave operators

$$
U^{ \pm}=\mathrm{s}-\lim _{t \rightarrow \mp \infty} \mathrm{e}^{\mathrm{i} t H} \mathrm{e}^{-\mathrm{i} t H_{0}} \mathbf{1}_{\Theta}\left(H_{0}\right)
$$

exist and are complete on $\Theta$.

Remark 1. This theorem is a variant of the well-known results concerning completeness of wave operators in Kato's theory of smooth perturbations (see e.g. Theorem XIII.31 in [RS2]).

Remark 2. For the above theorem to hold we do not need that either $H$ or $H_{0}$ have purely absolutely continuous spectrum on $\Theta$.

Proof. We first show that

$$
\mathrm{S}-\lim _{t \rightarrow \mp \infty} \mathrm{e}^{\mathrm{i} t H} \mathbf{1}^{\mathrm{ss}} \mathrm{e}^{-\mathrm{i} t H_{0}} \mathbf{1}_{\Theta}\left(H_{0}\right)=0 .
$$

Let $\psi \in \operatorname{Ran} \mathbf{1}_{\Theta}\left(H_{0}\right)$ and

$$
\ell(t):=\mathrm{e}^{\mathrm{i} t H_{0}} \mathbf{1}^{\mathrm{ss}} \mathrm{e}^{-\mathrm{i} t H_{0}} \psi
$$

Obviously,

$$
\left\|\mathrm{e}^{\mathrm{i} t H} \mathbf{1}^{\mathrm{ss}} \mathrm{e}^{-\mathrm{i} t H_{0}} \psi\right\|=\|\ell(t)\| .
$$

By (a), the function $\ell(t)$ is square-integrable and by $(c)$ its derivative is uniformly bounded. These two facts yield that $\lim _{|t| \rightarrow \infty} \ell(t)=0$ (see e.g. Exercise 62 in [RS2]).

We now show that the limits

$$
\mathrm{s}-\lim _{t \rightarrow \mp \infty} \mathbf{1}_{\Theta}(H) \mathrm{e}^{\mathrm{i} t H} \mathbf{1}^{\mathrm{bb}} \mathrm{e}^{-\mathrm{i} t H_{0}} \mathbf{1}_{\Theta}\left(H_{0}\right)
$$

exist. Let $\psi \in \mathfrak{H}$ be given and let

$$
w(t):=\mathbf{1}_{\Theta}(H) \mathrm{e}^{\mathrm{i} t H} \mathbf{1}^{\mathrm{bb}} \mathrm{e}^{-\mathrm{i} t H_{0}} \mathbf{1}_{\Theta}\left(H_{0}\right) \psi .
$$

Then for any $\phi \in \mathfrak{H}$,

$$
\frac{\mathrm{d}}{\mathrm{d} t}(\phi \mid w(t))=\mathrm{i}\left(V \mathbf{1}_{\Theta}(H) \mathrm{e}^{-\mathrm{i} t H} \phi \mid T V \mathrm{e}^{-\mathrm{i} t H_{0}} \mathbf{1}_{\Theta}\left(H_{0}\right) \psi\right),
$$

where we used the assumption (b). Therefore, if $t>s$,

$$
|(\phi \mid w(t)-w(s))| \leq\|T\|\left(\int_{s}^{t}\left\|V \mathbf{1}_{\Theta}(H) \mathrm{e}^{-\mathrm{i} \tau H} \phi\right\|^{2} \mathrm{~d} \tau\right)^{\frac{1}{2}}\left(\int_{s}^{t}\left\|V \mathbf{1}_{\Theta}\left(H_{0}\right) \mathrm{e}^{-\mathrm{i} \tau H_{0}} \psi\right\|^{2} \mathrm{~d} \tau\right)^{\frac{1}{2}} .
$$


It follows from Proposition 2.7 that for some constant $C_{1}$,

$$
\int_{\mathbf{R}}\left\|V \mathbf{1}_{\Theta}(H) \mathrm{e}^{-\mathrm{i} \tau H} \phi\right\|^{2} \mathrm{~d} \tau \leq C_{1}\|\phi\|^{2} .
$$

Thus, for some constant $C$,

$$
\|w(t)-w(s)\| \leq C\left(\int_{s}^{t}\left\|V \mathbf{1}_{\Theta}\left(H_{0}\right) \mathrm{e}^{-\mathrm{i} t H_{0}} \psi\right\|^{2} \mathrm{~d} t\right)^{\frac{1}{2}} .
$$

By the assumption (b), the integrand on the right hand side of the last equation is in $L^{1}(\mathbf{R})$. Therefore, the sequence $w(t)$ is Cauchy as $t \rightarrow \infty$ and $t \rightarrow-\infty$ and the limits (2.20) exist.

Let $I=[a, b]$ be an interval contained in $\Theta$ and let $\gamma$ be a simple closed curve in the complex plane that separates $[a, b]$ and $\mathbf{R} \backslash \Theta$ and encloses $[a, b]$. Let $\mathcal{R}(z)=(H-z)^{-1}$, $\mathcal{R}_{0}(z)=\left(H_{0}-z\right)^{-1}$. Then for any $\psi \in \mathcal{H}$,

$$
\begin{aligned}
& \mathbf{1}_{\mathbf{R} \backslash \Theta}(H) \mathrm{e}^{\mathrm{i} t H} \mathbf{1}^{\mathrm{bb}} \mathrm{e}^{-\mathrm{i} t H_{0}} \mathbf{1}_{I}\left(H_{0}\right) \psi= \\
& =(2 \pi \mathrm{i})^{-1} \oint_{\gamma} \mathbf{1}_{\mathbf{R} \backslash \Theta}(H) \mathrm{e}^{\mathrm{i} t H}\left(\mathcal{R}(z) \mathbf{1}^{\mathrm{bb}}-\mathbf{1}^{\mathrm{bb}} \mathcal{R}_{0}(z)\right) \mathrm{e}^{-\mathrm{i} t H_{0}} \mathbf{1}_{I}\left(H_{0}\right) \psi \mathrm{d} z \\
& =-(2 \pi \mathrm{i})^{-1} \oint_{\gamma} \mathbf{1}_{\mathbf{R} \backslash \Theta}(H) \mathrm{e}^{\mathrm{i} t H} \mathcal{R}(z)\left(H \mathbf{1}^{\mathrm{bb}}-\mathbf{1}^{\mathrm{bb}} H_{0}\right) \mathcal{R}_{0}(z) \mathrm{e}^{-\mathrm{i} t H_{0}} \mathbf{1}_{I}\left(H_{0}\right) \psi \mathrm{d} z .
\end{aligned}
$$

It follows that for some constant $C$,

$$
\left\|\mathbf{1}_{\mathbf{R} \backslash \Theta}(H) \mathrm{e}^{\mathrm{i} t H} \mathbf{1}^{\mathrm{bb}} \mathrm{e}^{-\mathrm{i} t H_{0}} \mathbf{1}_{I}\left(H_{0}\right) \psi\right\| \leq C \oint_{\gamma}\left\|V \mathcal{R}_{0}(z) \mathrm{e}^{-\mathrm{i} t H_{0}} \mathbf{1}_{I}\left(H_{0}\right) \psi\right\| \mathrm{d} z .
$$

Let

$$
l(z, t):=V \mathcal{R}_{0}(z) \mathrm{e}^{-\mathrm{i} t H_{0}} \mathbf{1}_{I}\left(H_{0}\right) \psi .
$$

The function $l(z, t)$ is uniformly bounded on $\gamma \times \mathbf{R}$ and has a uniformly bounded derivative in $t$. Moreover, by the assumption (b), for all $z \in \gamma, l(z, t)$ is square-integrable in $t$. It follows that $\lim _{|t| \rightarrow \infty} l(z, t)=0$. This implies that

$$
\mathrm{s}-\lim _{t \rightarrow \mp \infty} \mathbf{1}_{\mathbf{R} \backslash \Theta}(H) \mathrm{e}^{\mathrm{i} t H} \mathbf{1}^{\mathrm{bb}} \mathrm{e}^{-\mathrm{i} t H_{0}} \mathbf{1}_{I}\left(H_{0}\right)=0,
$$

and since $\Theta$ is a countable union of closed intervals, that

$$
\mathrm{S}-\lim _{t \rightarrow \mp \infty} \mathbf{1}_{\mathbf{R} \backslash \Theta}(H) \mathrm{e}^{\mathrm{i} t H} \mathbf{1}^{\mathrm{bb}} \mathrm{e}^{-\mathrm{i} t H_{0}} \mathbf{1}_{\Theta}\left(H_{0}\right)=0 .
$$

This fact, (2.19) and the existence of limits (2.20) imply that the wave operators $U^{ \pm}$exist.

To prove that the wave operators $U^{ \pm}$are complete on $\Theta$ it suffices to show that the wave operators

$$
\tilde{U}^{ \pm}:=\mathrm{s}-\lim _{t \rightarrow \mp \infty} \mathrm{e}^{\mathrm{i} t H_{0}} \mathrm{e}^{-\mathrm{i} t H} \mathbf{1}_{\Theta}(H)
$$

exist. However, since (b) implies that $H_{0} \mathbf{1}^{\mathrm{bb}}-\mathbf{1}^{\mathrm{bb}} H=-V T^{*} V$, the proof of the existence of $\tilde{U}^{ \pm}$is the same as the proof of the existence of $U^{ \pm}$. 


\subsection{The Feshbach formula and the resonant spectrum}

In this section we consider the operators $H$ on $\mathfrak{H}=\mathfrak{H}^{\mathrm{s}} \oplus \mathfrak{H}^{\mathrm{b}}$ of the form

$$
H=\left[\begin{array}{ll}
H^{\mathrm{ss}} & H^{\mathrm{sb}} \\
H^{\mathrm{bs}} & H^{\mathrm{bb}}
\end{array}\right],
$$

where $H^{\text {ss }}$ and $H^{\mathrm{bb}}$ are self-adjoint operators on $\mathfrak{H}^{\mathrm{s}}$ and $\mathfrak{H}^{\mathrm{b}}, H^{\mathrm{bs}}: \mathfrak{H}^{\mathrm{s}} \mapsto \mathfrak{H}^{\mathrm{b}}$ is a bounded operator and $\left(H^{\mathrm{bs}}\right)^{*}=H^{\mathrm{sb}}$. Clearly, $H$ is a self-adjoint operator and $\mathcal{D}(H)=\mathcal{D}\left(H^{\mathrm{ss}}\right) \oplus$ $\mathcal{D}\left(H^{\mathrm{bb}}\right)$.

For any $z \notin \sigma\left(H^{\mathrm{bb}}\right)$ we define

$$
\begin{aligned}
& W_{\mathrm{s}}(z):=H^{\mathrm{sb}}\left(H^{\mathrm{bb}}-z \mathbf{1}^{\mathrm{bb}}\right)^{-1} H^{\mathrm{bs}}, \\
& G_{\mathrm{s}}(z):=H^{\mathrm{ss}}-W_{\mathrm{s}}(z)-z \mathbf{1}^{\mathrm{ss}} .
\end{aligned}
$$

We will call $G_{\mathrm{s}}(z)$ the resonance function. Note that for $e \in \mathbf{R} \backslash \sigma\left(H^{\mathrm{bb}}\right)$ the operator $G_{\mathrm{s}}(e)$ is selfadjoint and that $G_{\mathrm{s}}(z)$ is an analytic family of type A on $\mathbf{C} \backslash \sigma\left(H^{\mathrm{bb}}\right)$.

The following result is known as the Feshbach formula. For the proof we refer the reader to [DJ], Proposition 3.5.

Proposition 2.2 Assume that $z \notin \sigma\left(H^{\mathrm{bb}}\right)$. Then,

(i) $z \notin \sigma(H)$ iff $0 \notin \sigma\left(G_{\mathrm{s}}(z)\right)$.

(ii) If $0 \notin \sigma\left(G_{\mathrm{s}}(z)\right)$ then

$$
\begin{aligned}
(H-z)^{-1} & \left.=\left(\mathbf{1}^{\mathrm{ss}}-\left(H^{\mathrm{bb}}-z \mathbf{1}^{\mathrm{bb}}\right)^{-1} H^{\mathrm{bs}}\right)\right) G_{\mathrm{s}}^{-1}(z)\left(\mathbf{1}^{\mathrm{ss}}-H^{\mathrm{sb}}\left(H^{\mathrm{bb}}-z \mathbf{1}^{\mathrm{bb}}\right)^{-1}\right) \\
& +\left(H^{\mathrm{bb}}-z \mathbf{1}^{\mathrm{bb}}\right)^{-1} .
\end{aligned}
$$

The spectral consequences of the Feshbach formula are discussed in detail in [DJ]. On one occasion we will make use of the following result (Theorem 3.6 in [DJ]).

Theorem 2.3 Assume that $e \in \mathbf{R} \backslash \sigma\left(H^{\mathrm{bb}}\right)$. Then $e$ is an eigenvalue of $H$ iff 0 is an eigenvalue of $G_{\mathrm{s}}(e)$. Moreover, $\operatorname{dim} \mathbf{1}_{\{e\}}(H)=\operatorname{dim} \mathbf{1}_{\{0\}}\left(G_{\mathrm{s}}(e)\right)$.

We make the following hypothesis:

(A1) For any $e \in \mathbf{R}$, the norm-limit

$$
\lim _{y \downarrow 0} W_{\mathrm{s}}(e+\mathrm{i} y)=: W_{\mathrm{s}}(e)
$$

exists, and the function $\overline{\mathbf{C}}_{+} \ni z \mapsto W_{\mathrm{s}}(z)$ is norm-continuous. 
Notation. It is common to denote the value of the limit $(2.23)$ by $W_{\mathrm{s}}(e+\mathrm{i} 0)$. Since we will never deal with the boundary values $\lim _{y \uparrow 0} W_{\mathrm{s}}(e+\mathrm{i} y)$, for notational simplicity we write $e$ for $e+\mathrm{i} 0$.

Until the end of this section we assume that Hypothesis (A1) holds.

We set

$$
G_{\mathrm{s}}(e):=H^{\mathrm{ss}}-W_{\mathrm{s}}(e)-e \mathbf{1}^{\mathrm{ss}}, \quad e \in \mathbf{R} .
$$

$G_{\mathrm{s}}(e)$ is a closed operator and $\mathcal{D}\left(G_{\mathrm{s}}(e)\right)=\mathcal{D}\left(H^{\mathrm{ss}}\right)$. We define the resonant spectrum of the operator $H$ by the formula

$$
\mathfrak{R}(H):=\left\{e \in \mathbf{R}: 0 \in \sigma\left(G_{\mathrm{s}}(e)\right)\right\} .
$$

\section{Proposition 2.4}

(i) $\mathfrak{R}(H)$ is a closed set.

(ii) $\mathfrak{R}(H) \subset \sigma(H)$.

(iii) $\sigma(H) \backslash \sigma\left(H^{\mathrm{bb}}\right) \subset \mathfrak{R}(H)$.

Proof. Part (iii) is an immediate consequence of Proposition 2.2.

We will prove Part (i) by showing that the set $\mathbf{R} \backslash \mathfrak{R}(H)$ is open. Let $e_{0} \in \mathbf{R} \backslash \mathfrak{R}(H)$ be given. Then, it follows from Hypothesis (A1) that there exists $\delta>0$ such that

$$
\sup _{\left|e-e_{0}\right|<\delta}\left\|\left(G_{\mathrm{S}}(e)-G_{\mathrm{S}}\left(e_{0}\right)\right) G_{\mathrm{S}}^{-1}\left(e_{0}\right)\right\|<1 .
$$

For $e \in\left(e_{0}-\delta, e_{0}+\delta\right)$ we write

$$
G_{\mathrm{s}}(e)=T(e) G_{\mathrm{s}}\left(e_{0}\right),
$$

where

$$
T(e)=1+\left(G_{\mathrm{s}}(e)-G_{\mathrm{s}}\left(e_{0}\right)\right) G_{\mathrm{s}}^{-1}\left(e_{0}\right) .
$$

It follows from (2.24) that $T(e)$ is a bounded, invertible operator. Relation (2.25) yields that $G_{\mathrm{s}}(e)$ is invertible and that $G_{\mathrm{s}}^{-1}(e)=G_{\mathrm{s}}^{-1}\left(e_{0}\right) T(e)^{-1}$. Therefore,

$$
\left(e_{0}-\delta, e_{0}+\delta\right) \subset \mathbf{R} \backslash \mathfrak{R}(H),
$$

and Part (i) follows.

To prove Part (ii), note first that for $z \in \mathbf{C}_{+}$the Feshbach formula yields

$$
\mathbf{1}^{\mathrm{ss}}(H-z)^{-1} \mathbf{1}^{\mathrm{ss}}=G_{\mathrm{s}}^{-1}(z) .
$$

Therefore, if $e \notin \sigma(H)$ then the function $\mathbf{C}_{+} \ni z \mapsto G_{\mathrm{S}}^{-1}(z)$ has an analytic extension to a neighborhood of $e$. We denote this extension by $Q(z)$. Clearly, for $z \in \mathbf{C}_{+}$and any $\psi, G_{\mathrm{s}}(z) Q(z) \psi=\psi$ (in particular, $\operatorname{Ran} Q(z)=\mathcal{D}\left(H^{\mathrm{ss}}\right)$ ). Moreover, it follows from Hypothesis $(\mathrm{A} 1)$ that $\left(G_{\mathrm{s}}(z)-G_{\mathrm{s}}(e)\right) Q(z) \rightarrow 0$ as $z \rightarrow e$. Thus, for any $\psi, G_{\mathrm{s}}(e) Q(z) \psi \rightarrow$ $\psi$ as $z \rightarrow e$. Since $G_{\mathrm{s}}(e)$ is a closed operator, we conclude that for any $\psi, Q(e) \psi \in$ $\mathcal{D}\left(G_{\mathrm{s}}(e)\right)$ and $G_{\mathrm{s}}(e) Q(e) \psi=\psi$. It follows from the closed graph theorem that $0 \notin$ $\sigma\left(G_{\mathrm{s}}(e)\right)$.

The next observation we need is 
Lemma 2.5 The function

$$
\overline{\mathbf{C}}_{+} \backslash \mathfrak{R}(H) \ni z \mapsto G_{\mathrm{s}}^{-1}(z),
$$

with values in bounded operators on $\mathfrak{H}^{\mathrm{s}}$, is continuous in the norm topology.

Proof. Let $z_{0} \in \overline{\mathbf{C}}_{+} \backslash \mathfrak{R}(H)$ be given. It follows from Hypothesis (A1) that there exists $\delta>0$ such that if $\left|z-z_{0}\right|<\delta$ and $z \in \overline{\mathbf{C}}_{+}$then

$$
\left\|\left(G_{\mathrm{s}}(z)-G_{\mathrm{s}}\left(z_{0}\right)\right) G_{\mathrm{s}}^{-1}\left(z_{0}\right)\right\|<1 / 2 .
$$

If in addition $z \notin \mathfrak{R}(H)$, this relation and the identity

$$
G_{\mathrm{s}}^{-1}(z)-G_{\mathrm{s}}^{-1}\left(z_{0}\right)=G_{\mathrm{s}}^{-1}(z)\left(G_{\mathrm{s}}\left(z_{0}\right)-G_{\mathrm{s}}(z)\right) G_{\mathrm{s}}^{-1}\left(z_{0}\right)
$$

yield that

$$
G_{\mathrm{s}}^{-1}(z)=G_{\mathrm{s}}^{-1}\left(z_{0}\right)\left(1-\left(G_{\mathrm{s}}(z)-G_{\mathrm{s}}\left(z_{0}\right)\right) G_{\mathrm{s}}^{-1}\left(z_{0}\right)\right)^{-1} .
$$

In particular, we get that

$$
\left\|G_{\mathrm{s}}^{-1}(z)\right\| \leq 2\left\|G_{\mathrm{s}}^{-1}\left(z_{0}\right)\right\| .
$$

Going back to the equation (2.27) we derive that if $z \in \overline{\mathbf{C}}_{+} \backslash \mathfrak{R}(H)$ and $\left|z-z_{0}\right|<\delta$, then

$$
\begin{aligned}
\left\|G_{\mathrm{s}}^{-1}(z)-G_{\mathrm{s}}^{-1}\left(z_{0}\right)\right\| & \leq 2\left\|G_{\mathrm{s}}^{-1}\left(z_{0}\right)\right\|^{2}\left\|G_{\mathrm{s}}(z)-G_{\mathrm{s}}\left(z_{0}\right)\right\| \\
& =2\left\|G_{\mathrm{s}}^{-1}\left(z_{0}\right)\right\|^{2}\left\|W_{\mathrm{s}}(z)-W_{\mathrm{s}}\left(z_{0}\right)+\left(z-z_{0}\right) \mathbf{1}^{\mathrm{ss}}\right\| .
\end{aligned}
$$

The result follows from this relation and Hypothesis (A1).

The following technical result will be used on several occasions in this paper.

Proposition 2.6 Let $T$ be a closed operator of the form $T=A+\mathrm{i} B$, where $A$ and $B$ are self-adjoint operators and $B$ is bounded. Assume further that $B \leq 0$. Then,

(i) $\operatorname{Ker} T=\operatorname{Ker} A \cap \operatorname{Ker} B$.

(ii) If $0 \in \sigma(T)$ then $0 \in \sigma(A) \cap \sigma(B)$.

(iii) If $0 \in \sigma(T)$ and $y>0$ then $\left\|(T+\mathrm{i} y)^{-1}\right\|=y^{-1}$.

Proof. Let $\psi \in \operatorname{Ker} T$. Then $(\psi \mid T \psi)=(\psi \mid A \psi)+\mathrm{i}(\psi \mid B \psi)=0$ which yields $(\psi \mid B \psi)=0$. Since $B \leq 0, B \psi=0$ and $A \psi=(T-\mathrm{i} B) \psi=0$. Therefore, $\operatorname{Ker} T \subset \operatorname{Ker} A \cap \operatorname{Ker} B$. The inclusion $\supset$ is obvious, and Part (i) follows.

To establish Part (ii), we note first that $\mathcal{D}(T)=\mathcal{D}\left(T^{*}\right)=\mathcal{D}(A)$ and that the numerical range of $T$ is contained in $\overline{\mathrm{C}}_{-}$. By the numerical range theorem (see e.g. Lemma 4.5 in [JP]) we have that $\sigma(T) \subset \overline{\mathbf{C}}_{-}$. Therefore, if $0 \in \sigma(T)$ then 0 is on the topological boundary of $\sigma(T)$. It follows (see e.g. [VH], Theorem 2.1) that there exists a sequence $\psi_{n},\left\|\psi_{n}\right\|=1$, such that

$$
\lim _{n} T \psi_{n}=0 .
$$


Now, (2.28) yields that $\lim _{n}\left(\psi_{n} \mid B \psi_{n}\right)=0$, and since $B \leq 0$, that $\lim _{n} B \psi_{n}=0$. This and (2.28) yield that $\lim _{n} A \psi_{n}=0$. Thus, $\psi_{n}$ is Weyl's sequence of $A$ and $B$ and $0 \in$ $\sigma(A) \cap \sigma(B)$.

Finally, to prove (iii), let $\psi_{n}$, with $\left\|\psi_{n}\right\|=1$, be such that $\lim _{n} T \psi_{n}=0$. Set $\phi_{n}:=$ $T \psi_{n}$. Then

$$
\psi_{n}=(T+\mathrm{i} y)^{-1} \phi_{n}+\mathrm{i} y(T+\mathrm{i} y)^{-1} \psi_{n}
$$

which yields that

$$
\left\|(T+\mathrm{i} y)^{-1}\right\| \geq y^{-1}-\left\|(T+\mathrm{i} y)^{-1} \phi_{n}\right\| .
$$

Letting $n \rightarrow \infty$ we conclude that $\left\|(T+\mathrm{i} y)^{-1}\right\| \geq y^{-1}$. Since the numerical range theorem implies that $\left\|(T+\mathrm{i} y)^{-1}\right\| \leq y^{-1}$, (iii) follows.

Our next result is

Proposition $2.7 e \in \sigma(H) \backslash \mathfrak{R}(H)$ iff there exists $\delta>0$ such that

$$
\sup _{x \in(e-\delta, e+\delta), y>0}\left\|\mathbf{1}^{\mathrm{ss}}(H-x-\mathrm{i} y)^{-1} \mathbf{1}^{\mathrm{ss}}\right\|<\infty .
$$

In particular, the projection $\mathbf{1}^{\mathrm{ss}}$ is $H$-smooth on any compact subset of $\sigma(H) \backslash \mathfrak{R}(H)$.

Proof. If $e \in \sigma(H) \backslash \mathfrak{R}(H)$ then the relation (2.29) follows from Lemmas 2.4 and 2.5.

Assume now that $e \in \mathfrak{R}(H)$. Since

$$
\left(G_{\mathrm{S}}(e)+\mathrm{i} y\right)^{-1}=G_{\mathrm{s}}^{-1}(e+\mathrm{i} y)\left(1-\left(W_{\mathrm{s}}(e+\mathrm{i} y)-W_{\mathrm{s}}(e)\right)\left(G_{\mathrm{s}}(e)+\mathrm{i} y\right)^{-1}\right)
$$

and

$$
\left\|\left(G_{\mathrm{S}}(e)+\mathrm{i} y\right)\right\|^{-1}=y^{-1}
$$

(we used Proposition 2.6), it follows that

$$
\left\|G_{\mathrm{s}}^{-1}(e+\mathrm{i} y)\right\| \geq(y+l(y))^{-1},
$$

where $l(y):=\left\|W_{\mathrm{s}}(e+\mathrm{i} y)-W_{\mathrm{s}}(e)\right\|$. By Lemma 2.5, $\lim _{y \downarrow 0} l(y)=0$, and (2.30) implies that for $e \in \mathfrak{R}(H)$ the relation (2.29) does not hold for any $\delta>0$. This yields the statement.

The last proposition does not imply that the spectrum of $H$ on the set $\sigma(H) \backslash \mathfrak{R}(H)$ is purely absolutely continuous. Indeed, the fact that $\mathbf{1}^{\text {ss }}$ is $H$-smooth on any compact subset of $\sigma(H) \backslash \mathfrak{R}(H)$ implies only that

$$
\operatorname{Ran}\left(\mathbf{1}_{\sigma(H) \backslash \mathfrak{R}(H)}(H) \mathbf{1}^{\mathrm{ss}}\right) \subset \mathfrak{H}_{\mathrm{ac}}(H) .
$$

(see Theorem XIII.23 in [RS2]). To establish that the spectrum of $H$ on $\sigma(H) \backslash \mathfrak{R}(H)$ is purely absolutely continuous we need an additional assumption on our abstract model. We recall that a set $\Gamma$ is called cyclic for $H$ if the linear span of the set $\{g(H) \psi: g \in$ $\left.C_{\infty}(\mathbf{R}), \psi \in \Gamma\right\}$ is dense in $\mathfrak{H}$. Our hypothesis is:

(A2) The surface Hilbert space $\mathfrak{H}^{\mathrm{s}}$ is a cyclic set for $H$. 
Proposition 2.8 Assume that $\mathrm{A}(2)$ holds. Then the spectrum of $H$ on the set $\sigma(H) \backslash$ $\mathfrak{R}(H)$ is purely absolutely continuous.

Proof. By (2.31), for any $g \in C_{\infty}(\mathbf{R}), g(H) \mathbf{1}_{\sigma(H) \backslash \mathfrak{R}(H)}(H) \mathbf{1}^{\mathrm{ss}} \subset \mathfrak{H}_{\text {ac }}(H)$. This observation and Hypothesis (A1) imply that $\operatorname{Ran} \mathbf{1}_{\sigma(H) \backslash \mathfrak{R}(H)}(H) \subset \mathfrak{H}_{\mathrm{ac}}(H)$.

\section{Deterministic results}

\subsection{Basic facts}

In this section we establish a number of technical results which will be used throughout the paper, we verify Hypotheses (A1)-(A2) of Section 2 and prove Proposition 1.2 and Theorem 1.3.

Let $\mathbf{T}=\mathbf{R} / 2 \pi \mathbf{Z}$ be the circle and $\mathbf{T}^{d}$ the $d$-dimensional torus. We denote the points in $\mathbf{T}^{d}$ by $\phi=\left(\phi_{1}, \ldots, \phi_{d}\right)$, and by $\mathrm{d} \phi$ the usual Lebesgue measure. In the sequel we identify $\mathcal{H}^{\mathrm{s}}$ with $l^{2}\left(\mathbf{Z}^{d}\right)$. Let $\mathcal{F}: l^{2}\left(\mathbf{Z}^{d}\right) \mapsto L^{2}\left(\mathbf{T}^{d}\right)$ be the usual Fourier transform,

$$
(\mathcal{F} \psi)(\phi)=(2 \pi)^{-\frac{d}{2}} \sum_{n \in \mathbf{Z}^{d}} \psi(n) \mathrm{e}^{\mathrm{i} n \cdot \phi} .
$$

In the sequel we will use the shorthand $\Phi(\phi):=2 \sum_{k=1}^{d} \cos \phi_{k}$. For $z \in \mathbf{C} \backslash \sigma\left(H_{0}\right)$, let $\lambda(\phi, z)$ be the solution of the quadratic equation

$$
X+X^{-1}+\Phi(\phi)=z
$$

which satisfies $|\lambda(\phi, z)|<1$. This solution also satisfies $\pm \operatorname{Im} \lambda(\phi, z)<0$ if $z \in \mathbf{C}_{ \pm}$(write $\lambda$ in the polar form).

We adopt the shorthand $\mathcal{R}(z)=(H-z)^{-1}$, and, for $\mathbf{m}, \mathbf{n} \in \mathbf{Z}_{+}^{d+1}$, we set

$$
\mathcal{R}(\mathbf{m}, \mathbf{n} ; z):=\left(\delta_{\mathbf{m}} \mid(H-z)^{-1} \delta_{\mathbf{n}}\right) .
$$

Let

$$
\hat{\mathcal{R}}((m, 0),(\phi, x) ; z)=(2 \pi)^{-\frac{d}{2}} \sum_{n \in \mathbf{Z}^{d}} \mathcal{R}((m, 0),(n, x) ; z) \mathrm{e}^{\mathrm{i} n \phi} .
$$

We set $\mathcal{R}_{0}(z)=\left(H_{0}-z\right)^{-1}$ and define $\mathcal{R}_{0}(\mathbf{m}, \mathbf{n} ; z)$ and $\hat{\mathcal{R}}_{0}((m, 0),(\phi, x) ; z)$ analogously.

Proposition 3.1 Let $x \geq 0$ and $m \in \mathbf{Z}^{d}$ be given. Then,

(i) $\hat{\mathcal{R}}((m, 0),(\phi, x) ; z)=\hat{\mathcal{R}}((m, 0),(\phi, 0) ; z) \lambda(\phi, z)^{x}$.

(ii) $\hat{\mathcal{R}}_{0}((m, 0),(\phi, x) ; z)=-(2 \pi)^{-\frac{d}{2}} \mathrm{e}^{\mathrm{i} m \phi} \lambda(\phi, z)^{x+1}$. 
Proof. For fixed $\mathbf{m}$ the matrix elements (3.33) satisfy the equation

$$
\begin{aligned}
\mathcal{R}(\mathbf{m},(n, x+1) ; z)+ & \mathcal{R}(\mathbf{m},(n, x-1) ; z)+\sum_{\left|n-n^{\prime}\right|_{+}=1} \mathcal{R}\left(\mathbf{m},\left(n^{\prime}, x\right) ; z\right) \\
& =\delta_{\mathbf{m n}}+z \mathcal{R}(\mathbf{m},(n, x) ; z),
\end{aligned}
$$

if $x>0$, and

$$
\mathcal{R}(\mathbf{m},(n, 1) ; z)+\sum_{\left|n-n^{\prime}\right|_{+}=1} R\left(\mathbf{m},\left(n^{\prime}, 0 ; z\right)+(V(n)-z) \mathcal{R}(\mathbf{m},(n, 0) ; z)=\delta_{\mathbf{m n}}\right.
$$

if $x=0$. The first equation is equivalent to

$$
\hat{\mathcal{R}}(\mathbf{m},(\phi, x+1) ; z)+\hat{\mathcal{R}}(\mathbf{m},(\phi, x-1) ; z)+(\Phi(\phi)-z) \hat{\mathcal{R}}(\mathbf{m},(\phi, x) ; z)=0 .
$$

Of course, this equation is easily "integrated" and it follows that

$$
\hat{\mathcal{R}}(\mathbf{m},(\phi, x) ; z)=\hat{\mathcal{R}}(\mathbf{m},(\phi, 0) ; z) \lambda(\phi, z)^{x} .
$$

This yields Part (i).

If $V=0$ the equation (3.35) is equivalent to

$$
\hat{\mathcal{R}}_{0}(\mathbf{m},(\phi, 1) ; z)+(\Phi(\phi)-z) \hat{\mathcal{R}}_{0}(\mathbf{m},(\phi, 0) ; z)=(2 \pi)^{-\frac{d}{2}} \mathrm{e}^{\mathrm{i} m \phi} .
$$

Combining this relation with (3.37) we derive the equation

$$
\hat{\mathcal{R}}_{0}(\mathbf{m},(\phi, 0) ; z)(\lambda(\phi, z)+\Phi(\phi)-z)=(2 \pi)^{-\frac{d}{2}} \mathrm{e}^{\mathrm{i} m \phi},
$$

which yields that

$$
\hat{\mathcal{R}}_{0}(\mathbf{m},(\phi, 0) ; z)=-(2 \pi)^{-\frac{d}{2}} \mathrm{e}^{\mathrm{i} m \phi} \lambda(\phi, z) .
$$

Combining (3.39) with (3.37) we arrive at Part (ii).

For $z \in \mathbf{C} \backslash \sigma\left(H_{0}\right)$ the self-energy operators $W_{\mathrm{s}}(z)$ can be computed. We denote by $\lambda(z)$ the operator of multiplication by the function $\lambda(\phi, z)$. Then

Proposition $3.2 W_{\mathrm{s}}(z)=-\mathcal{F}^{-1} \lambda(z) \mathcal{F}$.

Proof. Note first that for any $n, m \in \mathbf{Z}^{d}$,

$$
\left(\delta_{(m, 0)} \mid H^{\mathrm{sb}}\left(H^{\mathrm{bb}}-z \mathbf{1}^{\mathrm{bb}}\right)^{-1} H^{\mathrm{bs}} \delta_{(n, 0)}\right)=\left(\delta_{(m, 0)} \mid\left(H_{0}-z\right)^{-1} \delta_{(n, 0)}\right) .
$$

This observation and Part (ii) of Proposition 3.1 yield

$$
\begin{aligned}
\left(\delta_{(m, 0)} \mid\left(H_{0}-z\right)^{-1} \delta_{(n, 0)}\right) & =(2 \pi)^{-\frac{d}{2}} \int_{\mathbf{T}^{d}} \hat{\mathcal{R}}_{0}(\mathbf{m},(\phi, 0) ; z) \mathrm{e}^{-\mathrm{i} n \phi} \mathrm{d} \phi \\
& =-(2 \pi)^{-d} \int_{\mathbf{T}^{d}} \lambda(\phi, z) \mathrm{e}^{\mathrm{i}(m-n) \phi} \mathrm{d} \phi \\
& =-(2 \pi)^{-d} \int_{\mathbf{T}^{d}} \lambda(\phi, z) \mathrm{e}^{\mathrm{i}(n-m) \phi},
\end{aligned}
$$


where we used that $\phi \mapsto \lambda(\phi, z)$ is an even function. Thus,

$$
\left(\delta_{(m, 0)} \mid\left(H_{0}-z\right)^{-1} \delta_{(n, 0)}\right)=-\left(\mathcal{F} \delta_{(m, 0)} \mid \lambda(z) \mathcal{F} \delta_{(n, 0)}\right) .
$$

Relations (3.40) and (3.41) yield the statement.

Proposition 3.3 The projection $\mathbf{1}^{\mathrm{ss}}$ is $H_{0}$-smooth.

Proof. For $z \in \mathbf{C}_{+}$,

$$
\left\|\mathbf{1}^{\mathrm{ss}}\left(H_{0}-z\right)^{-1} \mathbf{1}^{\mathrm{ss}}\right\|=\left\|W_{\mathrm{s}}(z)\right\|=\sup _{\phi \in \mathbf{T}^{d}}|\lambda(\phi, z)| \leq 1 .
$$

This yields the statement.

Note that the function $\lambda(\phi, z)$ is analytic in $\mathbf{T}^{d} \times\left(\mathbf{C} \backslash \sigma\left(H_{0}\right)\right)$ and that the operatorvalued function $\lambda(z)$ is analytic in $\mathbf{C} \backslash \sigma\left(H_{0}\right)$. We proceed to show that these functions have continuous extensions from $\mathbf{T}^{d} \times \mathbf{C}_{+}$to $\mathbf{T}^{d} \times \overline{\mathbf{C}}_{+}$and $\mathbf{C}_{+}$to $\overline{\mathbf{C}}_{+}$. For $e \in \mathbf{R}$ we define $\lambda(\phi, e)$ as the solution of the equation (3.32) which satisfies $|\lambda(\phi, e)| \leq 1, \operatorname{Im} \lambda(\phi, e) \leq 0$. Explicitly, for $z \in \overline{\mathbf{C}}_{+}$,

$$
\lambda(\phi, z)=\frac{1}{2}\left(\Phi(\phi)-z-\sqrt{(\Phi(\phi)-z)^{2}-4}\right),
$$

where the branch of the square root is fixed by $\operatorname{Im} \sqrt{w} \geq 0$ if $\operatorname{Im} w \geq 0$. If $w=x+\mathrm{i} y$, $y \geq 0$, this branch is given by

$$
\sqrt{w}=\frac{1}{\sqrt{2}}(\sqrt{|w|+x}+\mathrm{i} \sqrt{|w|-x})
$$

Thus, we clearly have

Lemma 3.4 The function $\mathbf{T}^{d} \times \overline{\mathbf{C}}_{+} \ni z \mapsto \lambda(\phi, z)$ is continuous.

For $e \in \mathbf{R}$ we set $W_{\mathrm{s}}(e):=-\mathcal{F}^{-1} \lambda(e) \mathcal{F}$. An immediate consequence of the previous lemma is

Lemma 3.5 The function $\overline{\mathbf{C}}_{+} \ni z \mapsto W_{\mathrm{s}}(z)$ is norm-continuous.

Proof. Since the function $\lambda(\phi, z)$ is continuous on $\mathbf{T}^{d} \times \overline{\mathbf{C}}_{+}$(and therefore uniformly continuous on $\mathbf{T}^{d} \times$ compacts), the statement follows from the formula

$$
\left\|W_{\mathrm{s}}\left(z_{1}\right)-W_{\mathrm{s}}\left(z_{2}\right)\right\|=\sup _{\phi \in \mathbf{T}^{d}}\left|\lambda\left(\phi, z_{1}\right)-\lambda\left(\phi, z_{2}\right)\right|
$$


The above results allow us to compute the resonance function $G_{\mathrm{s}}(z)$. For $z \in \mathbf{C} \backslash \sigma\left(H_{0}\right)$, we define the operator $h_{0}(z)$ on $\mathcal{H}^{\mathrm{s}}$ by

$$
h_{0}(z)=-\mathcal{F}^{-1} \lambda^{-1}(z) \mathcal{F} .
$$

Then,

$$
G_{\mathrm{s}}(z)=h_{0}(z)+V .
$$

For $e \in \sigma\left(H_{0}\right), G_{\mathrm{s}}(e)=h_{0}(e)+V$, where $h_{0}(e)=-\mathcal{F}^{-1} \lambda^{-1}(e) \mathcal{F}$. Note that the operator $G_{\mathrm{s}}(e)$ is self-adjoint iff $e \in \mathbf{R} \backslash \operatorname{int} \sigma\left(H_{0}\right)$.

For later reference we prove

Lemma 3.6 Let $n \in \mathbf{Z}^{d}$ and $e \in \operatorname{int} \sigma\left(H_{0}\right)$. Then

$$
\lim _{y \downarrow 0} \operatorname{Im}\left(\delta_{(n, 0)} \mid\left(H_{0}-e-\mathrm{i} y\right)^{-1} \delta_{(n, 0)}\right)=-\int_{\mathbf{T}^{d}} \operatorname{Im} \lambda(\phi, e) \mathrm{d} \phi>0 .
$$

Proof. That the limit exists and that the limit and the integral in (3.44) are equal follows from Lemmas 3.2, 3.4 and Relation (3.40). Because for $e \in \operatorname{int} \sigma\left(H_{0}\right)$ the set

$$
\{e:|\Phi(\phi)-e|<2\}
$$

has positive Lebesgue measure, the inequality in (3.44) follows from the definition of $\lambda(\phi, e)$.

Lemma 3.5 verifies Hypothesis (A1) of Section 2 for the model (1.1) with respect to decomposition (1.4).

We shall also need the following technical results which are slight generalizations of Lemmas 2.7 and 3.5. For $R \in \mathbf{Z}_{+}$let

$$
\Gamma_{R}:=\left\{(n, x): n \in \mathbf{Z}^{d}, 0 \leq x \leq R\right\},
$$

and let $\mathbf{1}_{R}$ be the orthogonal projection on $l^{2}\left(\Gamma_{R}\right)$. Clearly, $\mathbf{1}_{0}=\mathbf{1}^{\mathrm{ss}}$.

The next two results hold for an arbitrary $R$.

Lemma 3.7 The operator-valued function

$$
\mathbf{C}_{+} \ni z \mapsto \mathbf{1}_{R}\left(H_{0}-z\right)^{-1} \mathbf{1}_{R},
$$

extends by continuity to a continuous function on $\overline{\mathbf{C}}_{+}$. In particular, the projection $\mathbf{1}_{R}$ is $H_{0}$-smooth.

Proof. If $R=0$ then the result follows from Lemma 3.5. The case $R>0$ follows by induction from the Feshbach formula. $V$.

Let $V$ be surface potential and $H=H_{0}+V$. The next two propositions hold for all 
Proposition 3.8 The projection $\mathbf{1}_{R}$ is $H$-smooth on any compact subset of $\sigma(H) \backslash \mathfrak{R}(H)$.

Proof. If $R=0$ then the result follows from Lemma 2.7. The case $R>0$ follows from Lemma 3.7 and the Feshbach formula.

We now verify Hypothesis (A2).

Proposition 3.9 The surface Hilbert space $\mathcal{H}^{\mathrm{s}}$ is a cyclic set for $H$.

Proof. It suffices to show that the linear span of the vectors

$$
\left\{H^{m} \delta_{(n, 0)}: n \in \mathbf{Z}^{d}, m=0,1, \ldots\right\},
$$

is dense in $\mathcal{H}$. Since $V$ acts only along $\mathcal{H}^{\text {s }}$, to prove this fact it suffices to show that the linear span of the vectors

$$
\left\{H_{0}^{m} \delta_{(n, 0)}: n \in \mathbf{Z}^{d}, m=0,1, \ldots\right\}
$$

is dense in $\mathcal{H}$. To prove the last statement, note that

$$
H_{0} \delta_{(n, 0)}-\sum_{\left|n-n^{\prime}\right|_{+}=1} \delta_{\left(n^{\prime}, 0\right)}=\delta_{(n, 1)} .
$$

Thus, the vectors $\delta_{(n, 1)}, n \in \mathbf{Z}^{d}$, belong to the linear span of the set (3.46). An obvious induction yields the statement.

To summarize, all the assumptions of Section 2 hold for the model (1.1) with respect to the decomposition (1.3)-(1.5). In particular, Proposition 1.2 follows from Proposition 2.4. We finish this section with

Proof of Theorem 1.3. Let $T$ be a linear operator defined by

$$
T \delta_{(n, x)}=\left\{\begin{array}{cl}
-\delta_{(n, 1)} & \text { if } x=0 \\
\delta_{(n, 0)} & \text { if } x=1 \\
0 & \text { if } x>1
\end{array}\right.
$$

Note that $\|T\|=1$ and

$$
H \mathbf{1}^{\mathrm{bb}}-\mathbf{1}^{\mathrm{bb}} H_{0}=\left[H_{0}, \mathbf{1}^{\mathrm{bb}}\right]=T .
$$

Moreover, $T=\mathbf{1}_{1} T \mathbf{1}_{1}$ and $\left[H, \mathbf{1}^{\mathrm{ss}}\right]=\left[H_{0}, \mathbf{1}^{\mathrm{ss}}\right]$ is a bounded operator. Thus all the conditions of Theorem 2.1 are satisfied and Theorem 1.3 follows. 


\subsection{Wave operators}

In this section we prove Theorem 1.1.

Let $h$ be the usual discrete Laplacian on $l^{2}(\mathbf{Z})$ and $h_{D}$ the discrete Laplacian on $l^{2}\left(\mathbf{Z}_{+}\right)$ with Dirichlet boundary condition. The operator $h_{D}$ acts as follows:

$$
\left(h_{D} \psi\right)(n)=\psi(n+1)+\psi(n-1) \quad \text { if } n>0,
$$

and $\left(h_{D} \psi\right)(0)=\psi(1)$. We will use the shorthand $\langle x\rangle=\left(1+x^{2}\right)^{\frac{1}{2}}$.

Lemma 3.10 There is a dense set of vectors $\mathcal{T} \subset l^{2}(\mathbf{Z})$ such that for $\psi \in \mathcal{T}, n \in \mathbf{Z}$ and $k \geq 0$,

$$
\left|\left(\delta_{n} \mid \mathrm{e}^{-\mathrm{i} t h} \psi\right)\right|=C_{\psi, k}\langle t\rangle^{k}\langle n\rangle^{-k} .
$$

The constant $C_{\psi, k}$ does not depend on $n$.

Proof. In the Fourier representation,

$$
\left(\delta_{n} \mid \mathrm{e}^{-\mathrm{i} t h} \psi\right)=\frac{1}{2 \pi} \int_{\mathbf{T}} \mathrm{e}^{-\mathrm{i} n \phi} \mathrm{e}^{-2 \mathrm{i} t \cos \phi} \hat{\psi}(\phi) \mathrm{d} \phi .
$$

Let $\mathcal{T}$ be the set of all $\psi$ such that $\hat{\psi}$ is $C^{\infty}(\mathbf{T})$. Then the estimate (3.48) follows from integration by parts.

Lemma 3.11 There is a dense set of vectors $\mathcal{R} \subset l^{2}\left(\mathbf{Z}_{+}\right)$such that for $\psi \in \mathcal{R}, x \geq 0$ and $k \geq 0$,

$$
\left|\left(\delta_{x} \mid \mathrm{e}^{-\mathrm{i} t h_{D}} \psi\right)\right| \leq C_{\psi, x, k}\langle t\rangle^{-k}
$$

Proof. We define a unitary map $U: l^{2}\left(\mathbf{Z}_{+}\right) \mapsto l^{2}(\mathbf{Z})$ by

$$
(U \psi)(n)=\left\{\begin{array}{cl}
2^{-\frac{1}{2}} \psi(n) & \text { if } n \geq 0 \\
0 & \text { if } n=-1 \\
-2^{-\frac{1}{2}} \psi(-n-2) & \text { if } n<-1
\end{array}\right.
$$

Set $\tilde{l}:=U l^{2}\left(\mathbf{Z}_{+}\right)$. The Hilbert space $\tilde{l}$ is preserved by $h$ and $U h_{D} U^{-1}=h$. If $\mathcal{F}: l^{2}(\mathbf{Z}) \mapsto$ $L^{2}(\mathbf{T})$ is the usual Fourier transform, then the unitary map $W=\mathcal{F} U$ identifies $l^{2}\left(\mathbf{Z}_{+}\right)$ with the subspace $\tilde{L}$ of $L^{2}(\mathbf{T})$ which consists of the functions which satisfy

$$
f(-\phi)=-\mathrm{e}^{2 \mathrm{i} \phi} f(\phi)
$$

Moreover, $W h_{D} W^{-1}$ acts on this subspace as multiplication by $2 \cos \phi$. Thus, for any $\psi \in l^{2}\left(\mathbf{Z}_{+}\right)$,

$$
\left(\delta_{x} \mid \mathrm{e}^{-\mathrm{i} t h_{D}} \psi\right)=2^{-\frac{1}{2}}(2 \pi)^{-1} \int_{\mathbf{T}}\left(\mathrm{e}^{-\mathrm{i} x \phi}-\mathrm{e}^{\mathrm{i}(x-2) \phi}\right) \mathrm{e}^{-2 \mathrm{i} t \cos \phi}(W \psi)(\phi) \mathrm{d} \phi .
$$


Let $\tilde{\mathcal{R}}$ be the subspace of $\tilde{L}$ which consists of $C^{\infty}$ functions which satisfy (3.50) and which are equal to zero in neighbourhoods of 0 and $\pi$. Clearly, $\tilde{\mathcal{R}}$ is dense in $\tilde{L}$, and therefore $\mathcal{R}=W^{-1} \tilde{\mathcal{R}}$ is dense in $l^{2}\left(\mathbf{Z}_{+}\right)$. Moreover, it follows from (3.51) that Relation (3.49) holds for any $\psi \in \mathcal{R}$ (integrate by parts).

Proposition 3.12 There exists a dense set $\mathcal{D} \subset \mathcal{H}$ such that for $\psi \in \mathcal{D}, R \geq 0$ and $k \geq 0$

$$
\left\|\mathbf{1}_{R} \mathrm{e}^{-\mathrm{i} t H_{0}} \psi\right\|=O\left(\langle t\rangle^{-k}\right)
$$

Proof. Let $R$ be given. We take $\mathcal{D}=\mathcal{R} \otimes \mathcal{T} \otimes \ldots \otimes \mathcal{T}$, where $\mathcal{R}$ and $\mathcal{T}$ are as in Lemmas 3.10 and 3.11. The set $\mathcal{D}$ is dense in $l^{2}\left(\mathbf{Z}_{+}^{d+1}\right)$ and consists of finite linear combinations of the vectors

$$
f \otimes \psi_{1} \otimes \ldots \otimes \psi_{d}, \quad f \in \mathcal{R}, \psi_{k} \in \mathcal{T}
$$

Note that

$$
\left\|\mathbf{1}_{R} \mathrm{e}^{-\mathrm{i} t H_{0}} \psi\right\|^{2}=\sum_{0 \leq x \leq R} \sum_{n \in \mathbf{Z}^{d}}\left|\left(\delta_{(n, x)} \mid \mathrm{e}^{-\mathrm{i} t H_{0}} \psi\right)\right|^{2} .
$$

If $\psi$ has the form $(3.52)$ and $n=\left(n_{1}, \ldots, n_{d}\right)$, then

$$
\left(\delta_{(n, x)} \mid \mathrm{e}^{-\mathrm{i} t H_{0}} \psi\right)=\left(\delta_{x} \mid \mathrm{e}^{-\mathrm{i} t h_{D}} f\right) \prod_{j=1}^{d}\left(\delta_{n_{j}} \mid \mathrm{e}^{-\mathrm{i} t h} \psi_{j}\right) .
$$

It follows from Lemmas 3.10 and 3.11 that for any positive integers $k_{1}, k_{2}$ there exist constant $C_{\psi, x, k_{1}, k_{2}}$, which does not depend on $n$, such that

$$
\left|\left(\delta_{(n, x)} \mid \mathrm{e}^{-\mathrm{i} t H_{0}} \psi\right)\right| \leq C_{\psi, x, k_{1}, k_{2}}\langle t\rangle^{d k_{2}-k_{1}} \prod_{j=1}^{d}\left\langle n_{j}\right\rangle^{-k_{2}}
$$

Taking $k_{1}=2 d+2$ and $k_{2}=2$ we derive the result.

Proof of Theorem 1.1. We will consider only the case $t \rightarrow \infty$. It follows from Proposition 3.12 that

$$
\mathrm{S}-\lim _{t \rightarrow \infty} \mathbf{1}^{\mathrm{ss}} \mathrm{e}^{-\mathrm{i} t H_{0}}=0
$$

and so, by Cook's criterion, to prove the existence of $\Omega^{-}$it suffices to show that for a dense set of $\psi$ 's,

$$
\int_{0}^{\infty}\left\|\left(H \mathbf{1}^{\mathrm{bb}}-\mathbf{1}^{\mathrm{bb}} H_{0}\right) \mathrm{e}^{-\mathrm{i} t H_{0}} \psi\right\| \mathrm{d} t<\infty .
$$

By (3.47),

$$
\left\|\left(H \mathbf{1}^{\mathrm{bb}}-\mathbf{1}^{\mathrm{bb}} H_{0}\right) \mathrm{e}^{-\mathrm{i} t H_{0}} \psi\right\| \leq\left\|\mathbf{1}_{1} \mathrm{e}^{-\mathrm{i} t H_{0}} \psi\right\|,
$$

and (3.53) follows from Proposition 3.12. 


\subsection{The resonant spectrum}

In this section we prove Theorem 1.4.

Lemma 3.13 Let $A$ and $B$ be self-adjoint operators. Assume that $A$ is bounded and that $\sigma(A)$ is a connected set. Then $\sigma(A+B) \subset \sigma(A)+\sigma(B)$.

Proof. By adding a constant to $A$ we may assume that $\sigma(A)=[-\|A\|,\|A\|]$. Let $z$ be real and assume that $z \notin \sigma(A)+\sigma(B)$. Then $z \notin \sigma(B)$ and

$$
\left\|(z-B)^{-1} A\right\| \leq \frac{\|A\|}{\operatorname{dist}(z, \sigma(B))}<1 .
$$

The identity

$$
z-(A+B)=(z-B)\left(1+(z-B)^{-1} A\right),
$$

yields that $z \notin \sigma(A+B)$.

We recall that $\mathcal{S}_{\text {ext }}(V)$ is defined by (1.9) and that $h_{0}(e)$ is defined by (3.42).

Lemma $3.140 \in \sigma\left(\operatorname{Re} h_{0}(e)+V\right) \Rightarrow e \in \mathcal{S}_{\text {ext }}(V)$.

Proof. In the Fourier representation $\operatorname{Re} h_{0}(e)$ acts as operator of multiplication by $-\operatorname{Re} \lambda(\phi, e)^{-1}$. Since this function is continuous, $\sigma\left(\operatorname{Re} h_{0}(e)\right)$ is a connected set. Thus if $0 \in \sigma\left(\operatorname{Re} h_{0}(e)+V\right)$ then, by Lemma 3.13, $0 \in \sigma\left(\operatorname{Re} h_{0}(e)\right)+\sigma(V)$. Therefore, there exist $\phi_{0} \in \mathbf{T}^{d}$ and $a \in \sigma(V)$ such that

$$
\operatorname{Re} \lambda\left(\phi_{0}, e\right)^{-1}=a \text {. }
$$

If $|a| \geq 1$ then $\lambda\left(\phi_{0}, e\right)$ must be real, and we have that

$$
a+a^{-1}=\lambda\left(\phi_{0}, e\right)+\lambda\left(\phi_{0}, e\right)^{-1}=e-\Phi\left(\phi_{0}\right) .
$$

If $|a|<1$ then $\left|\lambda\left(\phi_{0}, e\right)\right|=1$ and

$$
2 a=\left(\lambda\left(\phi_{0}, e\right)+\lambda\left(\phi_{0}, e\right)^{-1}\right)=e-\Phi\left(\phi_{0}\right) .
$$

The identities (3.54) and (3.55) yield the statement.

We are now ready to finish the

Proof of Theorem 1.4. To prove Part (i) we have to show that

$$
\sigma(H) \backslash \sigma\left(H_{0}\right) \subset \mathcal{S}(V) \backslash \sigma\left(H_{0}\right) .
$$

First, we observe that $\sigma\left(H^{\mathrm{bb}}\right)=\sigma\left(H_{0}\right)$, so the Feshbach formula (Proposition 2.2) yields

$$
\left(\forall e \notin \sigma\left(H_{0}\right)\right) e \in \sigma(H) \Leftrightarrow 0 \in G_{\mathrm{S}}(e) .
$$


It follows from Lemma 3.13 that $\forall e \notin \sigma\left(H_{0}\right)$,

$$
\sigma\left(G_{\mathrm{s}}(e)\right) \subset \sigma\left(h_{0}(e)\right)+\sigma(V) .
$$

Thus, if $e \notin \sigma\left(H_{0}\right)$ and $0 \in \sigma\left(G_{\mathrm{s}}(e)\right)$ then for some $\phi_{0} \in \mathbf{T}^{d}$ and $a \in \sigma(V)$,

$$
\lambda\left(\phi_{0}, e\right)^{-1}=a .
$$

It follows that

$$
a+a^{-1}=\lambda\left(\phi_{0}, e\right)+\lambda\left(\phi_{0}, e\right)^{-1}=e-\Phi\left(\phi_{0}\right) .
$$

Therefore, $\forall e \notin \sigma\left(H_{0}\right), 0 \in \sigma\left(G_{\mathrm{s}}(e)\right) \Rightarrow e \in \mathcal{S}(V)$. This relation yields Part (i).

It follows from Proposition 2.6 that

$$
0 \in \sigma\left(G_{\mathrm{s}}(e)\right) \Rightarrow 0 \in \sigma\left(\operatorname{Re} G_{\mathrm{s}}(e)\right) .
$$

Since $\operatorname{Re} G_{\mathrm{s}}(e)=\operatorname{Re} h_{0}(e)+V$, Part (ii) of the theorem follows from Lemma 3.14. To prove Part (iii), we write

$$
G_{\mathrm{s}}(e)=h_{0}(e)\left(1+h_{0}^{-1}(e) V\right) .
$$

Thus, $0 \in \sigma\left(G_{\mathrm{s}}(e)\right)$ iff $-1 \in \sigma\left(h_{0}^{-1}(e) V\right)$. Since

$$
\left\|h_{0}^{-1}(e)\right\|=\sup _{\phi \in \mathbf{T}^{d}}|\lambda(\phi, e)| \leq 1,
$$

$\left\|h_{0}^{-1}(e) V\right\|<1$, and $0 \notin \sigma\left(G_{\mathrm{s}}(e)\right)$. This yields that $\mathfrak{R}(H)=\emptyset$.

\subsection{Repulsive surfaces}

Physically, the surface is repulsive if it repels the wave packets with energies in $\sigma\left(H_{0}\right)$. We make this heuristic notion mathematically precise by saying that the surface is repulsive if the set $\mathfrak{R}(H) \cap \sigma\left(H_{0}\right)$ has Lebesgue measure 0 .

In this section we will show that the surface is repulsive if the surface potential $V$ satisfies

$$
\lim _{|n| \rightarrow \infty} V(n)=0 \quad \text { or } \quad \lim _{|n| \rightarrow \infty}|V(n)|=\infty .
$$

Roughly, if $V \rightarrow 0, H_{0}+V$ is "close" to $H_{0}$, and the surface is repulsive due to the Dirichlet boundary condition. When $|V| \rightarrow \infty$, the surface is repulsive due to the force exerted by the surface potential. The goal of this section is to justify this rough picture.

First, we have

Proposition 3.15 Assume that (3.57) holds. Then $\sigma_{\mathrm{ess}}(H)=\sigma\left(H_{0}\right)$. 
Proof. If $V \rightarrow 0$, then $V$ is a compact operator, and the result follows from Weyl's theorem.

Assume that $|V| \rightarrow \infty$. Then the spectrum of $G_{\mathrm{s}}(z)=h_{0}(z)+V$ consists of a discrete set of eigenvalues of finite multiplicity which can accumulate only at $\pm \infty$. Assume that there exist $e_{0} \in \mathbf{R} \backslash \sigma\left(H_{0}\right)$ and a sequence $e_{k}$ such that $e_{k} \rightarrow e_{0}$ and $0 \in G_{\mathrm{s}}\left(e_{k}\right)$. Since $z \mapsto G_{\mathrm{s}}(z)$ is an analytic family of type $A$ in a neighborhood of $e_{0}$, by analytic perturbation theory (see e.g. [Ka], Chapter VII, Theorem 1.10), $0 \in \sigma\left(G_{\mathrm{s}}(z)\right)$ for $z$ in some neighborhood of $e_{0}$. However, for $z \in \mathbf{C}_{+}, \operatorname{Im} G_{\mathrm{s}}(z)<0$ and $0 \notin G_{\mathrm{s}}(z)$. Thus, the set

$$
E=\left\{e \in \mathbf{R}: e \notin \sigma\left(H_{0}\right), 0 \in \sigma\left(G_{\mathrm{s}}(e)\right)\right\},
$$

is discrete and $\pm 2(d+1), \pm \infty$ are its only possible accumulation points. By Proposition 2.2 and Theorem 2.3, the spectrum of $H$ outside $\sigma\left(H_{0}\right)$ is the set $E$, and each point of $E$ is an eigenvalue of $H$ of finite multiplicity. Therefore, the spectrum of $H$ outside $\sigma\left(H_{0}\right)$ is discrete.

The main result of this section is

Theorem 3.16 If $V$ is a surface potential which satisfies (3.57) then $\mathfrak{R}(H)$ has Lebesgue measure 0 .

An immediate consequence of this result and Theorem 1.3 is

Theorem 3.17 Let $V$ be a surface potential which satisfies (3.57). Let $\Theta \subset \sigma\left(H_{0}\right)$ be a Borel set such that the spectrum of $H$ on $\Theta$ is purely absolutely continuous. Then $\operatorname{Ran} \mathbf{1}_{\Theta}(H) \subset \operatorname{Ran} \mathbf{1}_{\sigma(H) \backslash \Re(H)}(H)$ and the wave operators $\Omega^{ \pm}$are complete on $\Theta$.

Remark. Theorem 1.7 follows from Theorems 1.6 and 3.17.

Before we prove Theorem 3.16, we recall

Proposition 3.18 Let $\overline{\mathbf{C}}_{+} \ni z \mapsto A(z)$ be a function with values in compact operators on a separable Hilbert space $\mathfrak{H}$. Assume that $A(z)$ is continuous in $\overline{\mathbf{C}}_{+}$and analytic in $\mathbf{C}_{+}$. Let $w \in \mathbf{C}$ be given. Then either $w \in \sigma(A(z))$ for all $z \in \overline{\mathbf{C}}_{+}$or the set $\{e \in \mathbf{R}: w \in \sigma(A(e))\}$ is a closed subset of $\mathbf{R}$ with Lebesgue measure 0 .

For the proof of this proposition we refer the reader to [RS1], Section XI.6.

Proof of Theorem 3.16. We recall that for $z \in \overline{\mathbf{C}}_{+}, G_{\mathrm{s}}(z)=h_{0}(z)+V$, and that the operator-valued functions $\overline{\mathbf{C}}_{+} \ni z \mapsto h_{0}(z), \overline{\mathbf{C}}_{+} \ni z \mapsto h_{0}^{-1}(z)$, are continuous in $\overline{\mathbf{C}}_{+}$and analytic in $\mathbf{C}_{+}$. Moreover, $\operatorname{Im} h_{0}(z)<0$ for $z \in \mathbf{C}_{+}$and $\lim _{\operatorname{Im} z \rightarrow \infty}\left\|h_{0}^{-1}(z)\right\|=0$.

Assume first that $\lim _{|n| \rightarrow \infty} V(n)=0$. Clearly, $V$ is a compact operator on $\mathcal{H}^{\mathrm{s}}$ and since

$$
G_{\mathrm{s}}(e)=h_{0}(e)\left(1+h_{0}^{-1}(e) V\right),
$$

$0 \in \sigma\left(G_{\mathrm{s}}(e)\right)$ iff $-1 \in \sigma\left(h_{0}^{-1}(e) V\right)$. For $z \in \overline{\mathbf{C}}_{+}$we set $A(z):=h_{0}^{-1}(z) V$. The function $A(z)$ satisfies all the conditions of Proposition 3.18 and $\lim _{\operatorname{Im} z \rightarrow \infty}\|A(z)\|=0$. Thus, 
if $\operatorname{Im} z$ is large enough, $-1 \notin \sigma(A(z))$ and it follows from Proposition 3.18 that the set $\{e \in \mathbf{R}:-1 \in \sigma(A(e))\}$ has Lebesgue measure 0 . This yields the statement.

Assume now that $\lim _{|n| \rightarrow \infty}|V(n)|=\infty$. Since

$$
G_{\mathrm{s}}(z)=(V+\mathrm{i})\left(1+(V+\mathrm{i})^{-1}\left(h_{0}(z)-\mathrm{i}\right)\right),
$$

$0 \in G_{\mathrm{s}}(z)$ iff $-1 \in \sigma\left((V+\mathrm{i})^{-1}\left(h_{0}(z)-\mathrm{i}\right)\right)$. Set $A(z):=(V+\mathrm{i})^{-1}\left(h_{0}(z)-\mathrm{i}\right)$. The function $A(z)$ satisfies all the conditions of Proposition 3.18. Since $|V| \rightarrow \infty$, the spectrum of $G_{\mathrm{s}}(z)$ consists of a discrete set of eigenvalues which can accumulate only at $\pm \infty$. Since $\operatorname{Im} h_{0}(z)<0$ if $\operatorname{Im} z>0$, this implies that $0 \notin G_{\mathrm{s}}(z)$ for $z \in \mathbf{C}_{+}$and so $-1 \notin \sigma(A(z))$. It follows from Proposition 3.18 that the set $\{e \in \mathbf{R}:-1 \in \sigma(A(e))\}$ has Lebesgue measure 0 and this yields the statement.

Finally, we discuss the case where $|V(n)|$ grows exponentially fast.

Proposition 3.19 Assume that for $n \in \mathbf{Z}^{d},|V(n)|>C_{1} \mathrm{e}^{\gamma|n|}+C_{2}$, where $C_{1}$ and $\gamma$ are positive constants. Then $\mathfrak{R}(H) \cap\left(-c_{d}, c_{d}\right)=\emptyset$.

Proof. Assume that $e \in\left(-c_{d}, c_{d}\right)$ and $0 \in \sigma\left(G_{\mathrm{s}}(e)\right)$. Then there exists a vector $\psi$, with $\|\psi\|=1$, such that $h_{0}(e) \psi+V \psi=0$. Since $V$ is increasing exponentially, $\psi$ is decaying exponentially and its Fourier transform $\hat{\psi}$ is an analytic function on $\mathbf{T}^{d}$. On the other hand, it follows from Proposition 2.6 that $\psi \in \operatorname{Ker} \operatorname{Im} h_{0}(e)$, and so $\hat{\psi}$ has to vanish on the set $\left\{\phi \in \mathbf{T}^{d}: \operatorname{Im} \lambda(\phi, e) \neq 0\right\}$. Since this set has positive Lebesgue measure, $\psi=0$.

\section{The i.i.d. random potentials}

In this section we prove Theorem 1.5.

Clearly, for any $V, \sigma\left(H_{0}\right) \subset \sigma(H)$, and since for i.i.d random variables $\mathcal{S}(V)=\mathcal{S}$ $P$-a.s., it follows from Theorem 1.4 that

$$
\Sigma \subset \sigma\left(H_{0}\right) \cup \mathcal{S}
$$

To show that $\mathcal{S} \subset \Sigma$ and to prove Part (ii) of Theorem 1.5 we need some additional facts.

Lemma 4.1 Let $a \in \operatorname{supp} \mu$ be given. Then, P-a.s. there exist a sequence of ( $V$ dependent) disjoint boxes

$$
B_{k}=\left\{n \in \mathbf{Z}^{d}:\left|n-c_{k}\right|_{+} \leq L_{k}\right\}
$$

where $\left|c_{k}\right|_{+} \rightarrow \infty, L_{k} \rightarrow \infty$, such that

$$
\forall n \in B_{k}, \quad|V(n)-a|<d_{k}
$$

where $d_{k} \rightarrow 0$. 
Proof. A standard application of the Borel-Cantelli lemma establishes the result.

Lemma 4.2 Let $V_{k}$ be a sequence of surface potentials such that $\forall n \in \mathbf{Z}^{d}, \lim _{k} V_{k}(n)=$ $V(n)$. Then $H_{0}+V_{k} \rightarrow H_{0}+V$ in the strong resolvent sense.

For any real constant $a$ we denote by $V_{a}$ the constant boundary potential $V_{a}(n)=a$. We use the shorthand $H_{a}=H_{0}+V_{a}$ and $\mathcal{S}_{a}=[-2 d, 2 d]+\left\{a+a^{-1}\right\}$

Lemma 4.3 Let $a,|a| \geq 1$, be given. Then,

(i) $\sigma\left(H_{a}\right)=\sigma\left(H_{0}\right) \cup \mathcal{S}_{a}$.

(ii) $\mathfrak{R}\left(H_{a}\right)=\mathcal{S}_{a}$.

The proofs of the last two lemmas are elementary and we will skip them.

Recall that for $j \in \mathbf{Z}^{d}$ the shift operator $T_{j}$ is defined by $\left(T_{j} V\right)(n)=V(n-j)$ and that $U_{j}$ is the unitary operator which acts as $\left(U_{j} \psi\right)(n, x)=\psi(n-j, x)$.

Proposition 4.4 There exists a set $\mathfrak{R}$ such that $\mathfrak{R}(H)=\mathfrak{R} P$-a.s.

Proof. Let $\mathfrak{B}(H)=\sigma(H) \backslash \mathfrak{R}(H)$. We will prove the equivalent statement, namely that there exist a set $\mathfrak{B}$ such that $\mathfrak{B}(H)=\mathfrak{B} P$-a.s. Let $H=H_{0}+V$ and, for $e \in \sigma(H)$, let

$$
\rho_{V}(e)=\lim _{\delta \downarrow 0} \sup _{x \in(e-\delta, e+\delta), y>0}\left\|\mathbf{1}^{\mathrm{ss}}(H-x-\mathrm{i} y)^{-1} \mathbf{1}^{\mathrm{ss}}\right\| .
$$

By Lemma 2.7, $e \in \mathfrak{B}(H)$ iff $\rho_{V}(e)<\infty$. It is not difficult to show that $\Omega \ni V \mapsto \rho_{V}(e)$ is a measurable function (see e.g. [CL]). Moreover, the relation (1.11) yields that for all $j, \rho_{T_{j} V}(e)=\rho_{V}(e)$. It follows (see e.g. Proposition 9.1 in $[\mathrm{CFKS}]$ ) that $\rho_{V}(e)$ is constant $P$-a.s. and so either $e \in \mathfrak{B}(H) P$-a.s. or $e \notin \mathfrak{B}(H) P$-a.s. Therefore, there exists a set $F \subset \mathbf{Q}$ such that $F=\mathbf{Q} \cap \mathfrak{B}(H) P$-a.s. Since $\mathfrak{B}(H)$ is a relatively open subset of $\sigma\left(H_{0}\right)$, the statement follows.

We say that a bounded operator $A$ on $\mathcal{H}$ is translationally invariant if for all $j, A U_{j}=$ $U_{j} A$.

Proposition 4.5 Let $A$ and $B$ be bounded, translationally invariant operators on $\mathcal{H}$ and let $a \in \operatorname{supp} \mu$ be given. Then for all $y \neq 0$ and $e \in \mathbf{R}$

$$
\left\|A(H-e-\mathrm{i} y)^{-1} B\right\| \geq\left\|A\left(H_{a}-e-\mathrm{i} y\right)^{-1} B\right\| .
$$

Proof. Let $\psi$ be a unit vector, let $V$ be given and let $B_{k}$ be the sequence of boxes from Lemma 4.1. Let $c_{k}$ be the center of $B_{k}$ and

$$
\psi_{k}:=U_{c_{k}} \psi, \quad V_{k}:=U_{c_{k}} V U_{-c_{k}}
$$


We write $H_{k}=H_{0}+V_{k}$. Since for every $n \in \mathbf{Z}^{d}, \lim _{k} V_{k}(n)=a, H_{k} \rightarrow H_{a}$ in the strong resolvent sense. This fact and the identity

$$
A(H-e-\mathrm{i} y)^{-1} B \psi_{k}=U_{-c_{k}} A\left(H_{k}-e-\mathrm{i} y\right)^{-1} \psi
$$

yield that

$$
\lim _{k \rightarrow \infty}\left\|A(H-e-\mathrm{i} y)^{-1} B \psi_{k}\right\|=\left\|A\left(H_{a}-e-\mathrm{i} y\right)^{-1} B \psi\right\|
$$

It follows that

$$
\left\|A(H-e-\mathrm{i} y)^{-1} B\right\| \geq\left\|A\left(H_{a}-e-\mathrm{i} y\right)^{-1} B \psi\right\| .
$$

Taking the supremum over $\psi$ we derive the statement.

Proof of Theorem 1.5. It remains to prove that $\mathcal{S} \subset \Sigma$ and $\mathcal{S} \subset \mathfrak{R}$. Let $a \in \operatorname{supp} \mu$, $|a| \geq 1$ be given and let $e \in \mathcal{S}_{a}$. Taking respectively $A=\mathbf{1}$ and $A=\mathbf{1}^{\mathrm{ss}}$ in Proposition 4.5 we deduce from this proposition and Lemmas 4.3 and 2.7 that $e \in \sigma(H)$ and $e \in \mathfrak{R}(H)$ $P$-a.s. Since $\sigma(H)=\Sigma$ and $\mathfrak{R}(H)=\mathfrak{R} P$-a.s., we derive that $\mathcal{S}_{a} \subset \Sigma$ and $\mathcal{S}_{a} \subset \mathfrak{R}$, and since

$$
\mathcal{S}=\bigcup_{a \in \operatorname{supp} \mu,|a| \geq 1} \mathcal{S}_{a}
$$

that $\mathcal{S} \subset \Sigma$ and $\mathcal{S} \subset \mathfrak{R}$.

\section{The absence of singular spectrum on $\sigma\left(H_{0}\right)$}

In this section we prove Theorem 1.6. Theorem 1.7 is an immediate consequence of Theorems 1.6 and 3.17 .

The proof of Theorem 1.6 consists of three technically and conceptually distinct steps. Step 1. Using Theorem 1.1 and general properties of the essential supports of subresolutions of the identity, we show that the essential support of the absolutely continuous spectrum of $H=H_{0}+V$ contains $\sigma\left(H_{0}\right)$ for any surface potential $V$.

Step 2. Using an argument based on the rank-one perturbation theory we show that $P$-a.s. the essential supports of the absolutely continuous components of the spectral measures of $\delta_{(n, 0)}$ for $H$ are equal for all $n$. It then follows from Step 1 and cyclicity of $\mathcal{H}^{\mathrm{s}}$ that $P$-a.s. these essential supports contain $\sigma\left(H_{0}\right)$.

Step 3. Parts 1 and 2 yield that for all $n \in \mathbf{Z}^{d}$, the singular spectrum of $H$, restricted to the cyclic subspace generated by $\delta_{(n, 0)}, P$-a.s. has Lebesgue measure zero on $\sigma\left(H_{0}\right)$. Spectral averaging (Simon-Wolff theorem) then yields that $P$-a.s. the operator $H$ has purely a.c. spectrum on $\sigma\left(H_{0}\right)$.

The principal novelty of our approach is Step 2 whose key technical ingredient is Theorem 5.9 below. In this step we were guided by an elegant argument of Simon [S3]. 


\section{$5.1 \quad$ Step 1}

We first recall several basic facts concerning various supports of Borel measures and subresolutions of the identity of a Hilbert space. Our discussion will follow closely [CL]. In the sequel, all Hilbert spaces are assumed to be separable.

Let $\mathcal{B}$ be the $\sigma$-algebra of Borel sets on $\mathbf{R}$ and $\mathfrak{H}$ a Hilbert space. A function $E$ on $\mathcal{B}$ with values in the space of projections on $\mathfrak{H}$ is called a subresolution of the identity of $\mathfrak{H}$ if the following holds:

(a) $E(\emptyset)=0$.

(b) For any sequence $A_{n}$ of disjoint Borel sets, $E\left(\cup A_{n}\right)=\sum E\left(A_{n}\right)$, where the series converges in the strong topology of $\mathfrak{H}$.

Notation. In the sequel, $\mathfrak{m}$ stands for Lebesgue measure on $\mathbf{R}$. As usual, we write $\mathrm{d} \mathfrak{m}=\mathrm{d} e$, and say simply a.e. $e$ for $\mathfrak{m}$-a.e. $e$.

Let $E$ be a subresolution of the identity of a Hilbert space $\mathfrak{H}$. We say that $E$ is supported on a Borel set $S$ if $E(\mathbf{R} \backslash S)=0$. The complement of the largest open set $B$ such that $E(B)=0$ is denoted by $\operatorname{supp} E$ and called the (topological) support of $E$. Somewhat less common in the literature is the notion of the essential support. An essential support of $E$ is a Borel set $\mathfrak{S}$ which satisfies the following:

(c) There exists Borel set $B_{0}$ with $\mathfrak{m}\left(B_{0}\right)=0$ such that $E$ is supported on $\mathfrak{S} \cup B_{0}$.

(d) For any Borel set $B, E(\mathfrak{S} \cap B)=0$ iff $\mathfrak{m}(\mathfrak{s} \cap B)=0$.

We will write $\mathfrak{S}=\operatorname{ess} \cdot \operatorname{supp} E$. Clearly, the essential support is defined up to a set of Lebesgue measure zero.

Let $\mu$ be a Borel measure on $\mathbf{R}$ and $\mathfrak{H}=L^{2}(\mathbf{R}, \mathrm{d} \mu)$. In the sequel, unless otherwise stated, all measures are assumed to be finite and nonnegative. We say that the measure $\mu$ is supported on a Borel set $S$ if $\mu(\mathbf{R} \backslash S)=0$. Let $E(B) f=\mathbf{1}_{B} f$, where $\mathbf{1}_{B}$ is the characteristic function of the Borel set $B$. Then $E$ is a subresolution of the identity of $\mathfrak{H}$, and its support and essential support are the support and the essential support of the measure $\mu$ (denoted respectively by $\operatorname{supp} \mu$ and ess.supp $\mu$ ). More explicitly, $\operatorname{supp} \mu$ is the complement of the largest open set such that $\mu(B)=0$, and the set $\mathfrak{S}$ is an essential support of $\mu$ if

(f) There exists a Borel set $B_{0}$ with $\mathfrak{m}\left(B_{0}\right)=0$ such that $\mu$ is supported on $\mathfrak{S} \cup B_{0}$.

(g) For any Borel set $B, \mu(\mathfrak{s} \cap B)=0$ iff $\mathfrak{m}(\mathfrak{S} \cap B)=0$.

Notation. To avoid repeating the phrase "up to a set of Lebesgue measure zero", in the sequel we write $B_{1} \subset B_{2}$ if the set $B_{1} \backslash B_{2}$ has Lebesgue measure zero. Thus, $B_{1}=B_{2}$ if the set $\left(B_{1} \backslash B_{2}\right) \cup\left(B_{2} \backslash B_{1}\right)$ has Lebesgue measure zero.

Let us state an obvious but useful fact.

Lemma 5.1 If $\mathrm{d} \mu=h(e) \mathrm{d} e$ then ess.supp $\mu=\{e: 0<h(e)<\infty\}$.

For our purposes, the Borel transform (sometimes called the Borel-Stieltjes or the Stieltjes transform) of a measure provides a convenient way to track its essential support. Let $\mu$ be a finite complex Borel measure on $\mathbf{R}$. Its Borel transform is defined for $z \in \mathbf{C}_{+}$ 
by

$$
G(z):=\int \frac{\mathrm{d} \mu(e)}{e-z} .
$$

The Borel transforms are studied in detail in [S2]. We summarize some results which we will need in the sequel.

Theorem 5.2 Assume that $\mu$ is a finite complex Borel measure on $\mathbf{R}$ and let $G(z)$ be its Borel transform. Then,

(i) The non-tangential limit

$$
\lim _{z \rightarrow e} G(z)=: G(e+\mathrm{i} 0),
$$

exists and is finite for a.e. $e \in \mathbf{R}$.

(ii) Assume that for some $z_{0} \in \mathbf{C}_{+}, G\left(z_{0}\right) \neq 0$. Then for all $w \in \mathbf{C}$ the set

$$
\{e \in \mathbf{R}: G(e+\mathrm{i} 0)=w\}
$$

has Lebesgue measure 0.

Proof. The measure $\mu$ can be written as a linear combination of the four positive measures. Thus, $G(z)$ can be written as a linear combination of the four Herglotz functions. For the Herglotz functions, however, the existence and finiteness of the non-tangential limit are well-known facts (see e.g. [Koo]).

Part (ii) is a consequence of the Lusin-Privalov theorem (see Section III.3 in [Koo]).

We can say more if $\mu$ is a Borel measure (hence finite and non-negative). Let $\mu_{\mathrm{ac}}, \mu_{\mathrm{pp}}$, $\mu_{\mathrm{sc}}$ be the absolutely continuous, pure point and singular continuous parts of $\mu$. We set $\mu_{\text {sing }}=\mu_{\mathrm{pp}}+\mu_{\mathrm{sc}}$.

Theorem 5.3 Let $\mu$ be a Borel measure and $G(z)$ its Borel transform. Then,

(i) For all $z \in \mathbf{C}_{+}, \operatorname{Im} G(z)>0$, so Part (ii) of Theorem 5.2 holds for $G(z)$.

(ii) The absolutely continuous component of $\mu$ is given by

$$
\mathrm{d} \mu_{\mathrm{ac}}=\pi^{-1} \operatorname{Im} G(e+\mathrm{i} 0) \mathrm{d} e .
$$

(iii) ess.supp $\mu_{\mathrm{ac}}=\{e: 0<\operatorname{Im} G(e+\mathrm{i} 0)<\infty\}$.

(iv) $\mu_{\text {sing }}$ is supported on $\left\{e: \lim _{y \downarrow 0} \operatorname{Im} G(e+\mathrm{i} y)=\infty\right\}$.

We now collect a few general results concerning the essential support of a subresolution of the identity.

Proposition 5.4 Let $\mathfrak{H}$ be a Hilbert space and $E$ a subresolution of the identity of $\mathfrak{H}$. Let $\left\{\psi_{k}, k \geq 1\right\}$ be an orthonormal basis of $\mathfrak{H}$. We define Borel measures $\mu_{k}$ and $\mu$ by

$$
\mu_{k}(B)=\left(\psi_{k} \mid E(B) \psi_{k}\right), \quad \mu(B)=\sum_{k=1}^{\infty} 2^{-k} \mu_{k}(B) .
$$


Then,

(i) ess.supp $\mu=\operatorname{ess} \cdot \operatorname{supp} E$.

(ii) ess.supp $\mu_{\mathrm{ac}}=\cup_{k=1}^{\infty}$ ess.supp $\mu_{k, \mathrm{ac}}$.

Proof. Part (i) is the exercise I.7.11 in [CL], so we prove only (ii).

Let $G$ be Borel transform of $\mu$ and $G_{k}$ Borel transform of $\mu_{k}$. Since

$$
\mu_{\mathrm{ac}}=\sum_{k=1}^{\infty} 2^{-k} \mu_{k, \mathrm{ac}}
$$

it follows from Theorem 5.3 that for a.e. $e$,

$$
\operatorname{Im} G(e+\mathrm{i} 0)=\sum_{k=1}^{\infty} 2^{-k} \operatorname{Im} G_{k}(e+\mathrm{i} 0)
$$

Since $\operatorname{Im} G_{k}(e+\mathrm{i} 0) \geq 0$,

$$
\{e: \operatorname{Im} G(e+\mathrm{i} 0)>0\}=\bigcup_{k=1}^{\infty}\left\{e: \operatorname{Im} G_{k}(e+\mathrm{i} 0)>0\right\} .
$$

Finally, since the sets $\{e: \operatorname{Im} G(e+\mathrm{i} 0)=\infty\},\left\{e: \operatorname{Im} G_{k}(e+\mathrm{i} 0)=\infty\right\}$ have Lebesgue measure zero, the result follows from Relation (5.59) and Theorem 5.3.

We will need

Lemma 5.5 Let $A$ be a self-adjoint operator on a Hilbert space $\mathfrak{H}$ and $\psi, \phi \in \mathfrak{H}$. Assume that $\phi$ is a cyclic vector for $A$, and let $\mu_{\psi}, \mu_{\phi}$ be the spectral measures of $\psi$ and $\phi$ for $A$. Then,

$$
\text { ess.supp } \mu_{\psi, \text { ac }} \subset \text { ess.supp } \mu_{\phi, \text { ac }} \text {. }
$$

Proof. $\mathfrak{H}$ is unitarily equivalent to $L^{2}\left(\mathbf{R}, \mathrm{d} \mu_{\phi}\right)$ so that $\phi=1$. The $L^{2}$-function associated to the vector $\psi$ will be denoted by $\psi(e)$. If $G$ is the Borel transform of $\mu_{\phi}$, then

$$
\mathrm{d} \mu_{\phi, \mathrm{ac}}=\operatorname{Im} G(e+\mathrm{i} 0) \mathrm{d} e, \quad \mathrm{~d} \mu_{\psi, \mathrm{ac}}=|\psi(e)|^{2} \operatorname{Im} G(e+\mathrm{i} 0) \mathrm{d} e,
$$

and the statement follows from Lemma 5.1.

Let $A$ be a self-adjoint operator on a Hilbert space $\mathfrak{H}$. The spectral theorem for $A$ yields that $\mathfrak{H}$ can be decomposed as

$$
\mathfrak{H}=\mathfrak{H}_{\mathrm{ac}} \oplus \mathfrak{H}_{\mathrm{pp}} \oplus \mathfrak{H}_{\mathrm{sc}}
$$

so that $A_{\mathrm{ac}}:=\left.A\right|_{\mathfrak{H}_{\mathrm{ac}}}$ has only absolutely continuous spectrum, $A_{\mathrm{pp}}:=\left.A\right|_{\mathfrak{H}_{\mathrm{pp}}}$ only pure point spectrum, $A_{\mathrm{sc}}:=\left.A\right|_{\mathfrak{H}_{\mathrm{sc}}}$ only singular continuous spectrum, and $A=A_{\mathrm{ac}} \oplus A_{\mathrm{pp}} \oplus A_{\mathrm{sc}}$. 
The spectral theorem further associates to $A$ a subresolution of the identity $E$ of $\mathfrak{H}$. Moreover,

$$
E=E_{\mathrm{ac}} \oplus E_{\mathrm{pp}} \oplus E_{\mathrm{sc}}
$$

where $E_{\mathrm{ac}}$ is the subresolution of the identity associated to $A_{\mathrm{ac}}$, etc. We also have that $\sigma(A)=\operatorname{supp} E, \sigma_{\mathrm{ac}}(A)=\operatorname{supp} E_{\mathrm{ac}}$, etc.

Proposition 5.6 Let $A$ be a self-adjoint operator on a Hilbert space $\mathfrak{H}$ and $E_{\mathrm{ac}}$ the subresolution of the identity associated to the absolutely continuous part of A. Assume that $\mathfrak{H}=\mathfrak{H}^{\mathrm{S}} \oplus \mathfrak{H}^{\mathrm{b}}$ and that $\mathfrak{H}^{\mathrm{S}}$ is a cyclic set for $A$. Let $\left\{\psi_{k}, k \geq 1\right\}$ be an orthonormal basis for $\mathfrak{H}^{\mathrm{s}}$, and $\mu_{k}$ the Borel measures defined by $\mu_{k}(B)=\left(\psi_{k} \mid E_{\mathrm{ac}}(B) \psi_{k}\right)$. Then

$$
\text { ess.supp } E_{\mathrm{ac}}=\bigcup_{k} \operatorname{ess} \cdot \operatorname{supp} \mu_{k}
$$

Proof. The relation

$$
\text { ess.supp } E_{\mathrm{ac}} \supset \bigcup_{k} \operatorname{ess} . \operatorname{supp} \mu_{k}
$$

follows from Proposition 5.4, so we have only to prove the inclusion $\subset$.

For notational simplicity we will assume that $\operatorname{dim} \mathfrak{H}^{\mathrm{s}}=\infty$. Let $\mathfrak{H}_{k}$ be the cyclic subspace generated by $\psi_{k}$. We set $k_{1}=1$ and $\mathcal{H}_{1}=\mathfrak{H}_{k_{1}}$. Assume that $\mathcal{H}_{j}$ and $k_{j}$ are defined for $j=1, \ldots, n-1$. We set

$$
\begin{gathered}
k_{n}=\min \left\{j: \mathfrak{H}_{j} \not \subset \oplus_{j=1}^{n-1} \mathcal{H}_{j}\right\}, \\
\mathcal{H}_{n}=\mathfrak{H}_{k_{n}} \ominus\left(\left(\oplus_{j=1}^{n-1} \mathcal{H}_{j}\right) \cap \mathfrak{H}_{k_{n}}\right) .
\end{gathered}
$$

Obviously, the subspaces $\mathcal{H}_{j}$ are mutually orthogonal and invariant under $A$. Moreover,

$$
\mathcal{H}_{j} \subset \mathfrak{H}_{k_{j}}, \quad \bigcup_{j=1}^{k_{n}} \mathfrak{H}_{j} \subset \oplus_{j=1}^{n} \mathcal{H}_{j} .
$$

It follows that the Hilbert space

$$
\mathcal{K}=\oplus_{j} \mathcal{H}_{j}
$$

contains $\mathfrak{H}^{\mathrm{S}}$ and is invariant under $A$, hence $\mathcal{K}$ contains the cyclic subspace generated by $\mathfrak{H}^{\mathrm{s}}$. Since $\mathfrak{H}^{\mathrm{s}}$ is a cyclic set for $A, \mathcal{K}=\mathfrak{H}$. It follows that there exists an orthonormal basis $\left\{\phi_{i}\right\}$ of $\mathfrak{H}$, so that each vector $\phi_{i}$ belong to some $\mathcal{H}_{j}$. Let $\eta_{i}$ be the Borel measures defined by $\eta_{i}(B)=\left(\phi_{i} \mid E_{\text {ac }}(B) \phi_{i}\right)$. Now, let $\phi_{i}$ be given and assume that $\phi_{i} \in \mathcal{H}_{j}$. Then, $\eta_{i}$ is an absolutely continuous measure, and by Lemma 5.5 and (5.60)

$$
\text { ess.supp } \eta_{i} \subset \text { ess.supp } \mu_{k_{j}} \text {. }
$$


It follows from Proposition 5.4 that

$$
\text { ess.supp } E_{\mathrm{ac}}=\bigcup_{i} \operatorname{ess} . \operatorname{supp} \eta_{i}
$$

therefore

$$
\text { ess.supp } E_{\mathrm{ac}} \subset \bigcup_{k} \operatorname{ess} . \operatorname{supp} \mu_{k}
$$

Proposition 5.6 allows us to complete the first step in the proof of Theorem 1.6.

Proposition 5.7 Let $V$ be an arbitrary surface potential, $H=H_{0}+V$, and $E_{\mathrm{ac}}$ the subresolution of the identity associated to absolutely continuous part of $H$. Let $\mu_{n}, n \in \mathbf{Z}^{d}$, be the spectral measures of $\delta_{(n, 0)}$ for $H$. Then,

(i) $\sigma\left(H_{0}\right) \subset$ ess.supp $E_{\text {ac }}$.

(ii) ess.supp $E_{\mathrm{ac}}=\cup_{n}$ ess.supp $\mu_{n, \mathrm{ac}}$.

Proof. It follows from Lemma 3.6 that for any $e \in \operatorname{int} \sigma\left(H_{0}\right)$ and $n \in \mathbf{Z}^{d}$,

$$
\lim _{y \downarrow 0} \operatorname{Im}\left(\delta_{(n, 0)} \mid\left(H_{0}-e-\mathrm{i} y\right)^{-1} \delta_{(n, 0)}\right)>0 .
$$

Therefore, by Lemma 5.4, the essential support of the subresolution of the identity associated to $H_{0}$ is $\sigma\left(H_{0}\right)$. Let $\Omega^{+}$be the wave operator from Theorem 1.1. The operator $H$ preserves $\operatorname{Ran} \Omega^{+}$and its restriction to $\operatorname{Ran} \Omega^{+}$is unitarily equivalent to $H_{0}$. Since the subresolutions of the identity associated to unitary equivalent operators have the same essential support, we derive that

$$
\sigma\left(H_{0}\right) \subset \operatorname{ess} \cdot \operatorname{supp} E_{\mathrm{ac}} .
$$

Part (ii) follows from Propositions 3.9 and 5.6.

\section{$5.2 \quad$ Step 2}

In this section we study the behavior of the essential support of a.c. measures under rank-one perturbations.

Theorem 5.8 Let $A$ be a self-adjoint operator on a Hilbert space $\mathfrak{H}$ and $\phi \in \mathfrak{H}$. Let

$$
A_{\lambda}=A+\lambda(\phi \mid \cdot) \phi, \quad \lambda \in \mathbf{R},
$$

and let $\mu_{\lambda}$ be the spectral measure of $\phi$ for $A_{\lambda}$. Then,

(i) For all $\lambda$, ess.supp $\mu_{\lambda, \text { ac }}=\operatorname{ess.supp} \mu_{0, a c}$.

(ii) Let $\eta$ be a Borel measure defined by

$$
\eta(B):=\int \frac{\mu_{\lambda}(B)}{1+\lambda^{2}} \mathrm{~d} \lambda .
$$

Then $\eta$ is mutually equivalent to Lebesgue measure. 
Proof. Part (ii) is a theorem of Simon-Wolff (Theorem 5 in [SW]), so we prove only Part (i).

Let $G_{\lambda}(z)$ be the Borel transform of $\mu_{\lambda}$. The resolvent identity yields

$$
G_{\lambda}(z)=\frac{G_{0}(z)}{1+\lambda G_{0}(z)}
$$

By Theorem 5.2, for a.e. $e \in \mathbf{R}$ the boundary values $G_{\lambda}(e+\mathrm{i} 0), G_{0}(e+\mathrm{i} 0)$ exist, are finite, $G_{0}(e+\mathrm{i} 0) \neq-\lambda^{-1}$, and

$$
\operatorname{Im} G_{\lambda}(e+\mathrm{i} 0)=\frac{\operatorname{Im} G_{0}(e+\mathrm{i} 0)}{\left|1+\lambda G_{0}(e+\mathrm{i} 0)\right|^{2}} .
$$

Therefore, for a.e. $e \in \mathbf{R}$,

$$
\operatorname{Im} G_{\lambda}(e+\mathrm{i} 0)>0 \quad \Leftrightarrow \quad \operatorname{Im} G_{0}(e+\mathrm{i} 0)>0 .
$$

This yields the statement.

Our next result is a generalization of Part (i) of the previous theorem.

Theorem 5.9 Let $A$ be a self-adjoint operator on a Hilbert space $\mathfrak{H}$ and let $\phi_{1}$, $\phi_{2}$ be two vectors in $\mathfrak{H}$. Assume that there exist complex numbers $z_{i j} \in \mathbf{C}_{+}$such that

$$
\left(\phi_{i} \mid\left(A-z_{i j}\right)^{-1} \phi_{j}\right) \neq 0 \quad \text { for } \quad i, j=1,2, i \neq j .
$$

Let

$$
A_{\lambda}:=A+\lambda\left(\phi_{1} \mid \cdot\right) \phi_{1}, \quad \lambda \in \mathbf{R},
$$

and let $\mu_{\lambda i}$ be the spectral measure of $\phi_{i}$ for $A_{\lambda}$. Then, for a.e. $\lambda$,

$$
\text { ess.supp } \mu_{\lambda 1, \text { ac }} \subset \text { ess.supp } \mu_{\lambda 2, \text { ac }} \text {. }
$$

Proof. For $z \in \mathbf{C}_{+}$we set

$$
G_{i j, \lambda}(z)=\left(\phi_{i} \mid\left(A_{\lambda}-z\right)^{-1} \phi_{j}\right) .
$$

When $\lambda=0$ we write simply $G_{i j}(z)$. By assumption (5.62), none of the functions $G_{i j}(z)$ is identically equal to zero. Therefore, by Theorem 5.2, the set

$$
\mathcal{S}_{i j}:=\left\{e: G_{i j}(e+\mathrm{i} 0)=0 \text { or }\left|G_{i j}(e+\mathrm{i} 0)\right|=\infty \text { or } \lim _{y \downarrow 0} G_{i j}(e+\mathrm{i} y) \text { does not exist }\right\},
$$

has Lebesgue measure 0 . 
The resolvent identity yields

$$
\begin{gathered}
G_{21, \lambda}(z)=\frac{G_{21}(z)}{1+\lambda G_{11}(z)} \\
G_{22, \lambda}(z)=G_{22}(z)-\lambda G_{21, \lambda}(z) G_{12}(z) .
\end{gathered}
$$

Substituting, we get

$$
G_{22, \lambda}(z)=G_{22}(z)-\lambda \frac{G_{21}(z) G_{12}(z)}{1+\lambda G_{11}(z)}
$$

Rearranging this identity, we derive

$$
\left|1+\lambda G_{11}(z)\right|^{2} G_{22, \lambda}(z)=\lambda^{2} a(z)+\lambda b(z)+c(z),
$$

where

$$
\begin{aligned}
a(z) & =\left|G_{11}(z)\right|^{2} G_{22}(z)-G_{11}^{*}(z) G_{21}(z) G_{12}(z) \\
b(z) & =G_{22}(z) \operatorname{Re} G_{11}(z)-G_{21}(z) G_{12}(z) \\
c(z) & =G_{22}(z)
\end{aligned}
$$

Let

$$
S:=\left\{e \in \mathbf{R} \backslash\left(\cup \mathcal{S}_{i j}\right): \operatorname{Im} G_{11}(e+\mathrm{i} 0)>0\right\} .
$$

If $e \in S$, setting $z=e+\mathrm{i} y$ in (5.65) and taking $y \downarrow 0$, we derive that

$$
\left|1+\lambda G_{11}(e+\mathrm{i} 0)\right|^{2} \operatorname{Im} G_{22, \lambda}(e+\mathrm{i} 0)=\lambda^{2} \operatorname{Im}(e+\mathrm{i} 0)+\lambda \operatorname{Im} b(e+\mathrm{i} 0)+\operatorname{Im} c(e+\mathrm{i} 0)
$$

Assume now that for $e \in S$ we have

$$
\operatorname{Im} G_{22, \lambda}(e+\mathrm{i} 0)=0, \quad \operatorname{Im} a(e+\mathrm{i} 0)=0, \quad \operatorname{Im} b(e+\mathrm{i} 0)=0 .
$$

It then follows from (5.68) and the third relation in (5.66) that

$$
\operatorname{Im} G_{22}(e+\mathrm{i} 0)=0 .
$$

Then, the second relation in (5.66) yields

$$
\operatorname{Im}\left(G_{21}(e+\mathrm{i} 0) G_{12}(e+\mathrm{i} 0)\right)=0 .
$$

The first relation in (5.66) and (5.71) yield

$$
\operatorname{Im} G_{11}(e+\mathrm{i} 0) \operatorname{Re}\left(G_{21}(e+\mathrm{i} 0) G_{12}(e+\mathrm{i} 0)\right)=0 .
$$

By the choice of $e, G_{21}(e+\mathrm{i} 0) G_{12}(e+\mathrm{i} 0) \neq 0$, and (5.71) implies that

$$
\operatorname{Re}\left(G_{21}(e+\mathrm{i} 0) G_{12}(e+\mathrm{i} 0)\right) \neq 0 \text {. }
$$


Thus, (5.72) implies that

$$
\operatorname{Im} G_{11}(e+\mathrm{i} 0)=0,
$$

which contradicts the choice of $e$. Therefore, if $\operatorname{Im} G_{22, \lambda}(e+\mathrm{i} 0)=0$ for some $e \in S$, we must have that either $a(e+\mathrm{i} 0) \neq 0$ or $b(e+\mathrm{i} 0) \neq 0$.

Let

$$
\begin{aligned}
& T:=\left\{(\lambda, e) \in \mathbf{R} \times S: \operatorname{Im} G_{22, \lambda}(e+\mathrm{i} 0)=0\right\}, \\
& P(e):=\left\{\lambda \in \mathbf{R}: \operatorname{Im} G_{22, \lambda}(e+\mathrm{i} 0)=0\right\}, \\
& Q(\lambda):=\left\{e \in S: \operatorname{Im} G_{22, \lambda}(e+\mathrm{i} 0)=0\right\} .
\end{aligned}
$$

Since $\operatorname{Im} G_{22, \lambda}(e+\mathrm{i} 0) \geq 0$, for fixed $e$ the function

$$
\lambda \mapsto \lambda^{2} \operatorname{Im} a(e+\mathrm{i} 0)+\lambda \operatorname{Im} b(e+\mathrm{i} 0)+\operatorname{Im} c(e+\mathrm{i} 0),
$$

is either identically equal to zero for all $\lambda$ or has at most one zero. If either $\operatorname{Im} a(e+\mathrm{i} 0) \neq 0$ or $\operatorname{Im} b(e+\mathrm{i} 0) \neq 0$, then clearly this function is not identically zero. Therefore, for $e \in S$ the set $P(e)$ consists of at most one point. By Fubini's theorem, the set $T$ has Lebesgue measure zero, and then, by Fubini's theorem again, for a.e. $\lambda$ the set $Q(\lambda)$ has Lebesgue measure zero. Therefore, for a.e. $\lambda$,

$$
\operatorname{Im} G_{22, \lambda}(e+\mathrm{i} 0)>0 \quad \text { for } \quad e \in S \backslash Q(\lambda) .
$$

Since $Q(\lambda)$ has Lebesgue measure zero, it follows that for a.e. $\lambda$,

$$
S \subset \operatorname{ess.supp} \mu_{\lambda 2, \mathrm{ac}} .
$$

On the other hand, by Lemma 5.8 and (5.67)

$$
\text { ess.supp } \mu_{\lambda 1, \mathrm{ac}}=S
$$

for all $\lambda$. The statement follows from (5.73) and (5.74).

We now proceed to verify that condition (5.62) of Theorem 5.9 holds for our model. To that end we need two lemmas.

Lemma 5.10 Let $A$ be self-adjoint operator on a Hilbert space $\mathfrak{H}$ and $\psi, \phi \in \mathfrak{H}$ two orthogonal vectors. Assume that $\phi \in \cap_{n>0} \mathcal{D}\left(A^{n}\right)$, and that

$$
\forall z \in \mathbf{C}_{+}, \quad\left(\psi \mid(A-z)^{-1} \phi\right)=0 .
$$

Then, for all $l \geq 0,\left(\psi \mid A^{l} \phi\right)=0$. 
Proof. We will prove by induction that the pair of statements

$$
\left(\psi \mid A^{l} \phi\right)=0 \quad \text { and } \quad \forall z \in \mathbf{C}_{+}, \quad\left(\psi \mid A^{l}(A-z)^{-1} \phi\right)=0,
$$

holds for all $l \geq 0$. Clearly, this is true for $l=0$. Assume that (5.75) holds for some $l$. Then, the identity

$$
z\left(\psi \mid A^{l}(A-z)^{-1} \phi\right)+\left(\psi \mid A^{l} \phi\right)=\left(\psi \mid A^{l+1}(A-z)^{-1} \phi\right),
$$

yields that

$$
\forall z \in \mathbf{C}_{+}, \quad\left(\psi \mid A^{l+1}(A-z)^{-1} \phi\right)=0 .
$$

Since

$$
\mathrm{s}-\lim _{\lambda \rightarrow \infty}-\mathrm{i} \lambda(A-\mathrm{i} \lambda)^{-1}=\mathbf{1},
$$

(5.76) implies that

$$
\left(\psi \mid A^{l+1} \phi\right)=0 .
$$

Our next lemma concerns the Dirichlet Laplacian $H_{0}$. For $\mathbf{n}=(n, x) \in \mathbf{Z}_{+}^{d}$ we set $|\mathbf{n}|_{+}=x+\sum_{j=1}^{d}\left|n_{j}\right|$. We denote by $\gamma_{\mathbf{n m}}$ the number of paths in $\mathbf{Z}_{+}^{d+1}$ of the length $|\mathbf{n}-\mathbf{m}|_{+}$which connect $\mathbf{n}$ and $\mathbf{m}$. Obviously, $\gamma_{\mathbf{n m}}>0$.

Lemma 5.11 Let $\mathbf{n}, \mathbf{m}$ be two sites in $\mathbf{Z}_{+}^{d+1}$. Then

$$
\left(\delta_{\mathbf{n}} \mid H_{0}^{l} \delta_{\mathbf{m}}\right)=\left\{\begin{array}{cl}
0 & \text { if } l<|\mathbf{m}-\mathbf{n}|_{+}, \\
\gamma_{\mathbf{n m}} & \text { if } l=|\mathbf{m}-\mathbf{n}|_{+} .
\end{array}\right.
$$

Proof. An elementary induction with respect to $k=|\mathbf{m}-\mathbf{n}|_{+}$.

We now verify the condition (5.62) of Theorem 5.9.

Proposition 5.12 Let $V$ be a surface potential, $H=H_{0}+V$, and $n, m \in \mathbf{Z}^{d}$. Then there exists $z \in \mathbf{C}_{+}$such that

$$
\left(\delta_{(n, 0)} \mid(H-z)^{-1} \delta_{(m, 0)}\right) \neq 0 .
$$

Proof. Clearly, we may assume that $n \neq m$. Assume that

$$
\forall z \in \mathbf{C}_{+}, \quad\left(\delta_{(n, 0)} \mid(H-z)^{-1} \delta_{(m, 0)}\right)=0 .
$$

Let $l=|m-n|_{+}$. Then, it follows from Lemmas 5.10 and 5.11 that

$$
\left(\delta_{(n, 0)} \mid H^{l} \delta_{(m, 0)}\right)=0, \quad\left(\delta_{(n, 0)} \mid H_{0}^{l} \delta_{(m, 0)}\right)=1 .
$$


Expanding $\left(H_{0}+V\right)^{l}$, we write $\left(\delta_{(n, 0)} \mid H^{l} \delta_{(m, 0)}\right)$ as a sum of the term

$$
\left(\delta_{(n, 0)} \mid H_{0}^{l} \delta_{(m, 0)}\right),
$$

and the terms

$$
\left(\delta_{(n, 0)} \mid H_{0}^{l_{1}} V^{l_{2}} \ldots H_{0}^{l_{2 k-1}} V^{l_{2 k}} \delta_{(m, 0)}\right),
$$

where $l_{j} \geq 0, \sum l_{j}=l$, and at least one of $l_{2 j}$ 's is bigger then zero. It follows from Lemma 5.11 that each of the terms (5.79) is zero. Therefore, the first identity in (5.78) yields that

$$
\left(\delta_{(n, 0)} \mid H_{0}^{l} \delta_{(m, 0)}\right)=0 .
$$

which of course contradicts the second identity in (5.78).

We will need the following measurability result. Recall that $\mathfrak{m}$ stands for the Lebesgue measure.

Proposition 5.13 Let $\mu_{n}$ be the spectral measure of $\delta_{(n, 0)}$ for $H=H_{0}+V$. Then for any $n, m \in \mathbf{Z}^{d}$, the function

$$
\Omega \ni V \mapsto \mathfrak{m}\left(\operatorname{ess.supp} \mu_{n, \mathrm{ac}} \backslash \operatorname{ess.supp} \mu_{m, \mathrm{ac}}\right)
$$

is measurable.

Proof. Let

$$
\chi_{n}=\left\{(V, e): 0<\lim _{l \rightarrow \infty} \operatorname{Im}\left(\delta_{(n, 0)} \mid\left(H-e-\mathrm{i} l^{-1}\right)^{-1} \delta_{(n, 0)}\right)<\infty\right\} .
$$

Since for every $l$ the function

$$
\Omega \times \mathbf{R} \ni(V, e) \mapsto\left(\delta_{(n, 0)} \mid\left(H-e-\mathrm{i} l^{-1}\right) \delta_{(n, 0)}\right),
$$

is $P \otimes \mathfrak{m}$ is measurable (see e.g. [CL]), the sets $\chi_{n}$ are measurable. Therefore, by Fubini's theorem, the function

$$
\Omega \ni V \mapsto \int_{\mathbf{R}}\left(1-\mathbf{1}_{\chi_{m}}(V, e)\right) \mathbf{1}_{\chi_{n}}(V, e) \mathrm{d} e=\mathfrak{m}\left(\operatorname{ess.supp} \mu_{n, \mathrm{ac}} \backslash \operatorname{ess} . \operatorname{supp} \mu_{m, \mathrm{ac}}\right)
$$

is measurable.

We are now ready to complete the second step in the proof of Theorem 1.6.

Proposition 5.14 Let $H$ be as in Theorem 1.6 and, for $n \in \mathbf{Z}^{d}$, let $\mu_{n}$ be the spectral measure of $\delta_{(n, 0)}$ for $H$. Let $E_{\mathrm{ac}}$ be the subresolution of the identity associated to absolutely continuous part of $H$. Then,

(i) $\forall n \in \mathbf{Z}^{d}$, ess.supp $\mu_{n, \text { ac }}=$ ess.supp $E_{\text {ac }} P-a . s$.

(ii) $\forall n \in \mathbf{Z}^{d}, \sigma\left(H_{0}\right) \subset$ ess.supp $\mu_{n, \text { ac }} P$-a.s. 
Proof. Part (ii) is an immediate consequence of (i) and Proposition 5.7. To prove (i), we fix two sites $n, m \in \mathbf{Z}^{d}$ and potential $V_{0} \in \Omega$ and consider the operator

$$
H_{\lambda}=H_{0}+V_{0}+\lambda\left(\delta_{(n, 0)} \mid \cdot\right) \delta_{(n, 0)} \cdot
$$

Let $\mu_{\lambda n}$ be the spectral measure of $\delta_{(n, 0)}$ for $H_{\lambda}$. Then, it follows from Theorem 5.9 that for a.e. $\lambda$,

$$
\text { ess.supp } \mu_{\lambda n, \text { ac }} \subset \text { ess.supp } \mu_{\lambda m, \text { ac }} \text {. }
$$

Since the random variable $V(n)$ has a density, it follows from Lemma 5.13 and Fubini's theorem that

$$
\int_{\Omega} \mathfrak{m}\left(\operatorname{ess.supp} \mu_{n, \mathrm{ac}} \backslash \text { ess.supp } \mu_{m, \mathrm{ac}}\right) \mathrm{d} P(V)=0 \text {. }
$$

Therefore,

$$
\mathfrak{m}\left(\text { ess.supp } \mu_{n, \mathrm{ac}} \backslash \text { ess.supp } \mu_{m, \mathrm{ac}}\right)=0 \quad P-\text { a.s. }
$$

and

$$
\text { ess.supp } \mu_{n, \text { ac }} \subset \text { ess.supp } \mu_{m, \text { ac }} \quad P-\text { a.s. }
$$

Reversing the roles of $n$ and $m$, we derive that the opposite inclusion also holds. Thus,

$$
\text { ess.supp } \mu_{n, \mathrm{ac}}=\text { ess.supp } \mu_{m, \mathrm{ac}} \quad P-\text { a.s. }
$$

Since by Proposition 5.7,

$$
\text { ess.supp } E_{\mathrm{ac}}=\bigcup_{n} \operatorname{ess} . \operatorname{supp} \mu_{n, \mathrm{ac}},
$$

we derive Part (i) from (5.80).

\section{$5.3 \quad$ Step 3}

We are now ready to complete the third step in our argument and finish the proof of Theorem 1.6. We will use the notation of Proposition 5.14. Fix $n \in \mathbf{Z}^{d}$ and let $V_{0} \in \Omega$ be such that

$$
\sigma\left(H_{0}\right) \subset \text { ess.supp } \mu_{n, \text { ac }}
$$

Here, $\mu_{n}$ is the spectral measure of $\delta_{(n, 0)}$ for $H=H_{0}+V_{0}$. Let $G_{n}$ be the Borel transform of $\mu_{n}$ and

$$
S_{n}:=\left\{e: 0<\operatorname{Im} G_{n}(e+\mathrm{i} 0)<\infty\right\} .
$$

(All these quantities depend on $V_{0}$.) Since $S_{n}=\operatorname{ess} . \operatorname{supp} \mu_{n}$, the set

$$
T_{n}:=\sigma\left(H_{0}\right) \backslash S_{n}
$$

has Lebesgue measure zero. 
Let

$$
H_{\lambda}=H_{0}+V_{0}+\lambda\left(\delta_{(n, 0)} \mid \cdot\right) \delta_{(n, 0)}, \quad \lambda \in \mathbf{R} .
$$

Let $\mu_{\lambda n}$ be the spectral measure of $\delta_{(n, 0)}$ for $H_{\lambda}$ and $G_{\lambda n}(z)$ its Borel transform. The relation

$$
G_{\lambda n}(z)=\frac{G_{n}(z)}{1+\lambda G_{n}(z)}
$$

yields that for all $\lambda$,

$$
\left\{e \in \sigma\left(H_{0}\right): \lim _{y \downarrow 0} \operatorname{Im} G_{\lambda n}(e+\mathrm{i} y)=\infty\right\} \subset T_{n} .
$$

Therefore, by Theorem 5.3, for all $\lambda$,

$$
\operatorname{supp} \mu_{\lambda n, \text { sing }} \subset T_{n}
$$

It follows from the Simon-Wolff theorem (recall Proposition 5.8) that the Borel measure

$$
\eta(B):=\int \frac{\mu_{\lambda n}(B)}{1+\lambda^{2}} \mathrm{~d} \lambda
$$

is equivalent to Lebesgue measure. Therefore, $\eta\left(T_{n}\right)=0$, and by $(5.83)$, for a.e. $\lambda$,

$$
\mu_{\lambda n}\left(T_{n}\right)=0
$$

This relation and (5.82) yield that for a.e. $\lambda$ the measure $\mu_{\lambda n}$ is absolutely continuous on $\sigma\left(H_{0}\right)$.

Let $\mathbf{1}_{\Theta}^{\text {sing }}(H)$ be the spectral projection of $H$ onto $\Theta$ associated to the singular spectrum. It is known (see e.g. [CL]) that the function

$$
\Omega \ni V \mapsto \mathbf{1}_{\Theta}^{\text {sing }}(H)
$$

is weakly measurable. Since the random variable $V(n)$ has density and Relation (5.81) holds $P$-a.s., it follows from Fubini's theorem that

$$
\int_{\Omega}\left(\delta_{(n, 0)} \mid \mathbf{1}_{\sigma\left(H_{0}\right)}^{\operatorname{sing}}(H) \delta_{(n, 0)}\right) \mathrm{d} P(V)=0
$$

Thus, $P$-a.s. the spectral measure $\mu_{n}$ is absolutely continuous on $\sigma\left(H_{0}\right)$. Therefore, the operator $H$ restricted to the cyclic subspace $\mathcal{H}_{n}$ generated by $\delta_{(n, 0)}$ has $P$-a.s. purely a.c. spectrum on $\sigma\left(H_{0}\right)$. By Lemma 3.9, the linear span of $\cup \mathcal{H}_{n}$ is dense in $\mathcal{H}$. It follows that the operator $H$ has $P$-a.s. purely a.c. spectrum on $\sigma\left(H_{0}\right)$. The proof of Theorem 1.6 is complete. 


\section{References}

[AM] Aizenman M., Molchanov S.: Localization at Large Disorder and at Extreme Energies: An Elementary Derivation. Commun. Math. Phys. 157, 245 (1993).

[AS] Avron J., Simon B.: Transient and reccurent spectrum. J. Func. Anal. 43, 1 (1981).

[BS] Boutet de Monvel A., Surkova A.: Localisation des états de surface pour une classe d'opérateurs de Schrodinger discrets à potentiels de surface quasi-périodiques. Helv. Phys. Acta, 71, 459 (1998).

[CS] Chahrour A., Sahbani J.: On the spectral and scattering theory of the Schrödinger operator with surface potential, preprint.

[CL] Carmona R., Lacroix J.: Spectral Theory of Random Schrödinger Operators. Birkhauser, Boston 1990.

[CFKS] Cycon H., Froese R., Kirsch W., Simon B.: Schrödinger Operators. SpringerVerlag, Berlin-Heidelberg 1987.

[G] Grinshpun V.: Localization for random potentials supported on a subspace. Lett. Math. Phys. 34, 103 (1995).

[DJ] Derezinski J., Jakšić V.: Spectral Theory of Pauli-Fierz Hamiltonians, preprint.

[DS] Davies E.B., Simon B.: Scattering Theory for Systems with Different Spatial Asymptotics on the Left and Right. Commun. Math. Phys. 63, 277 (1978).

[JL1] Jakšić V., Last Y.: Spectral Structure of Anderson Type Hamiltonians, submitted.

[JL2] Jakšić V., Last Y.: in preparation.

[JM1] Jakšić V., Molchanov S.: On the Spectrum of the Surface Maryland Model, Lett. Math. Phys. 45, 185 (1998).

[JM2] Jakšić V., Molchanov S.: On the Surface Spectrum in Dimension Two. Helv. Phys. Acta, 71, 629 (1999).

[JM3] Jakšić V., Molchanov S.: Localization of Surface Spectra, to appear in Commun. Math. Phys.

[JM4] Jakšić V., Molchanov S.: Wave Operators for the Surface Maryland Model, submitted. 
[JMP] Jakšić V., Molchanov S., Pastur L.: On the Propagation Properties of Surface Waves. Wave Propagation in Complex Media, IMA Vol. Math. Appl. 96, 143 (1998).

[JMP1] Jakšić V., Molchanov S., Pastur L.: in preparation.

[JP] Jakšić V., C.-A. Pillet.: On a model for quantum friction II: Dynamics and Fermi's Golden Rule at positive temperature. Commun. Math. Phys., 176, 619 (1996).

[Ka] Kato T.: Perturbation Theory for Linear Operators. Springer-Verlag, BerlinHeidelberg 1980.

[Koo] Koosis P.: Introduction to $H_{p}$ spaces. London Mathematical Society Lecture Note Series, 40, Cambridge University Press 1980.

[KP] Khoruzenko B.A., Pastur L.: The localization of surface states: an exactly solvable model. Physics Reports 288, 109-126 (1997).

[M] Molchanov S.: Lectures on Random Media. In Lectures on Probability, ed. P. Bernard, Lecture Notes in Mathematics, 1581, Springer-Verlag, Heidelberg 1994.

[MV1] Molchanov S., Vainberg B.: unpublished.

[MV2] Molchanov S., Vainberg B.: private communication.

[N] Naboko S.N.: Dense point spectra of Schrödinger and Dirac operators. Theor.math, 68, 18 (1986).

[P] Pastur L.: Surface waves: propagation and localization. Journées "Équations aux dérivées partielles" (Saint-Jean-de Monts, 1995), Exp. No. VI, Éxole Polytech. Palaisseau (1995).

[S1] Simon B.: Some Schrödinger operators with dense point spectrum. Proc. A.M.S. 125, 203 (1997).

[S2] Simon B.: Spectral Analysis of Rank One Perturbations and Applications. CRM Proc. Lecture Notes, 8, AMS, Providence, RI, 1995.

[S3] Simon B.: Cyclic vectors in the Anderson model. Rev. Math. Phys., 6, 1183 (1994).

[RS1] Reed, M., Simon, B.: Methods of Modern Mathematical Physics, III. Scattering Theory, London, Academic Press (1978).

[RS2] Reed M., Simon B.: Methods of Modern Mathematical Physics, IV. Analysis of Operators, London, Academic Press (1978). 
[SW] Simon B., Wolff T.: Singular Continuous Spectrum Under Rank One Perturbations and Localization for Random Hamiltonians. Commun. Pure Appl. Math. 39, 75 (1986).

[VH] Vock E., Hunziker W.: Stability of Schrödinger eigenvalue problems. Comm. Math. Phys., 83, 281 (1982). 